\title{
Accuracy assessments of cloud droplet size retrievals from polarized reflectance measurements by the research scanning polarimeter
}

\author{
Mikhail D. Alexandrov a,b,*, Brian Cairns ${ }^{b}$, Claudia Emde ${ }^{\mathrm{c}}$, \\ Andrew S. Ackerman ${ }^{\mathrm{b}}$, Bastiaan van Diedenhoven ${ }^{\mathrm{d}, \mathrm{b}}$ \\ a Department of Applied Physics and Applied Mathematics, Columbia University, 2880, Broadway, New York, NY 10025, USA \\ ${ }^{\mathrm{b}}$ NASA Goddard Institute for Space Studies, 2880 Broadway, New York, NY 10025, USA \\ c Meteorologisches Institut, Ludwig-Maximilians-Universit"at, Theresienstrasse 37, 80333, Munich, Germany \\ d Center for Climate Systems Research, Columbia University, 2880 Broadway, New York, NY 10025, USA
}

\section{A R T I C L E I N F O}

\section{Article history:}

Received 5 May 2012

Received in revised form 13 July 2012

Accepted 14 July 2012

Available online 9 August 2012

\section{Keywords:}

Electromagnetic scattering

Polarization

Mie theory

Rainbow

Optical particle characterization

Remote sensing

\begin{abstract}
A B S T R A C T
We present an algorithm for the retrieval of cloud droplet size distribution parameters (effective radius and variance) from the Research Scanning Polarimeter (RSP) measurements. The RSP is an airborne prototype for the Aerosol Polarimetery Sensor (APS), which was on-board of the NASA Glory satellite. This instrument measures both polarized and total reflectance in 9 spectral channels with central wavelengths ranging from 410 to $2260 \mathrm{~nm}$. The cloud droplet size retrievals use the polarized reflectance in the scattering angle range between $135^{\circ}$ and $165^{\circ}$, where they exhibit the sharply defined structure known as the rain- or cloud-bow. The shape of the rainbow is determined mainly by the single scattering properties of cloud particles. This significantly simplifies both forward modeling and inversions, while also substantially reducing uncertainties caused by the aerosol loading and possible presence of undetected clouds nearby. In this study we present the accuracy evaluation of our algorithm based on the results of sensitivity tests performed using realistic simulated cloud radiation fields.
\end{abstract}

(c) 2012 Elsevier Inc. All rights reserved.

\section{Introduction}

Understanding of cloud optical and microphysical properties and their influences on the interaction of clouds with solar radiation is essential for accurate climate projections. Cloud optical properties are determined by their liquid water content and droplet size distribution, which vary within clouds. Accurate and robust remote sensing estimates of droplet sizes for different cloud types, especially for broken clouds, are crucial for studies of indirect aerosol effects, which are focused on relationships between aerosols and cloud properties (such as cloud cover, height and particle size).

Satellite remote sensing methods in the solar spectral domain can use both total and polarized reflectance measurements for cloud droplet size retrievals. However, those currently operational are based on the multispectral measurements in absorbing and non-absorbing bands by e. g., the Moderate Resolution Imaging Spectroradiometer (MODIS), and do not include polarization (Nakajima \& King, 1990;

\footnotetext{
* Corresponding author at: Department of Applied Physics and Applied Mathematics, Columbia University, 2880, Broadway, New York, NY 10025, USA. Tel.: +1212678 5548; fax: + 12126785552 .

E-mail address: mda14@columbia.edu (M.D. Alexandrov).
}

Platnick et al., 2003). These retrievals have certain limitations owing to the influence of the 3D structure of clouds (especially broken cumulus and stratocumulus) on the reflected radiation, which is not accounted for in the 1D radiative transfer models used in the retrieval algorithms (Marshak et al., 2006; Zinner et al., 2010). In particular, Marshak et al. (2006) found that ignoring sub-pixel cloud variability produces a negative bias in the retrieved effective droplet radius, while ignoring cloud inhomogeneity at scales larger than a pixel scale, on the contrary, leads to overestimation of the average droplet size. The 3D shadowing and illumination effects also introduce dependence of the retrieval results on solar and viewing geometries. Vant-Hull et al. (2007) found that when 3D clouds are viewed near the backscatter geometry, the retrievals based on a plane-parallel model underestimate the effective radius, while the reverse is true when the satellite is far from the backscatter position. The satellite data survey by Girolamo et al. (2010) found that the retrieved cloud reflectance is indistinguishable from plane-parallel clouds in only $24 \%$ of cases, mostly limited to regions dominated by stratiform clouds at solar zenith angles less than $60^{\circ}$ (for other regions or solar angles this frequency drops sharply to as low as a few percent). Nakajima et al. (2010) found that the cloud droplet sizes retrieved from MODIS data would be strongly influenced by the vertical inhomogeneity of droplet size, hypothesizing the existence of small cloud droplets at cloud top as the reason for small droplet size retrievals using the 
3.7- $\mu \mathrm{m}$ channel and large drizzle drops influencing 2.1- and 1.6- $\mu \mathrm{m}$ channels. The sensitivity of MODIS particle size retrievals to drizzle formation was not, however, corroborated by Zinner et al. (2010). The gaseous (water vapor) absorption inside the cloud also creates uncertainties (positive bias) in retrievals of water cloud effective radius from total reflectance measurements (Platnick \& Valero, 1995) and a similar effect can result from the presence of an absorbing aerosol layer above the cloud (Coddington et al., 2010; Haywood et al., 2004).

Retrieval of cloud droplet size from polarized observations of the reflected light in the rainbow region (at scattering angles between $135^{\circ}$ and $165^{\circ}$ ) allows many of the uncertainties associated with 3D effects and unknown aerosol loadings to be avoided. This advantage results from the shape of the rainbow being dominated by single scattering of light by cloud particles, even though its amplitude can be affected by aerosols and the geometric structure of clouds. The dominance of single scattering as a determinant of the structure of the rainbow simplifies both forward modeling and inversions. The retrievals are just as accurate over land, or ocean (no surface albedo issues), and are valid independent of the optical depth down to unity (i.e. they work for common low water path clouds).

Another potential use of this technique is in combination with lidar measurements of cloud-top extinction. The polarimetric retrievals of effective radius and variance provide accurate estimates of the extinction cross-section of cloud droplets that, when divided into the extinction coefficient, allow the number concentration of cloud droplets to be determined. This quantity, unlike the optical characteristics, is directly related to aerosol effects on clouds (Brenguier et al., 2003). Hu et al. (2007) introduced a method for derivation of droplet number concentration from CALIPSO satellite lidar data combined with MODIS droplet size retrievals. Their approach used an empirical relationship between depolarization and cloud extinction coefficient (obtained using Monte Carlo simulations) and derived the extinction crosssection from the MODIS 3.7- $\mu \mathrm{m}$ channel size retrieval. However, since the MODIS dataset does not provide effective variance of the droplet size distribution there are non-negligible uncertainties in the estimate of extinction cross-section that affect the droplet number concentration. This obstacle would not affect a study where polarimetric retrievals of droplet size distribution are used.

The polarized rainbow technique has previously been used to retrieve cloud droplet effective radii from the Polarization and Directionality of the Earth's Reflectances (POLDER, Deschamps et al., 1994) measurements (Bréon \& Doutriaux-Boucher, 2005; Bréon \& Goloub, 1998). The inter-comparison study by Bréon and Doutriaux-Boucher (2005) found high correlation between POLDER and MODIS products over the oceans (with MODIS biased high by about $2 \mu \mathrm{m}$ ). This correlation, however, breaks down over most of the continental and polluted oceanic areas, where POLDER retrieves smaller droplets (less than $7 \mu \mathrm{m}$ effective radius).

While RSP allows both total and polarized reflectance methods to be used simultaneously, in this study we focus only on the polarized rainbow technique. Here we present the results of tests performed on the output from 1D and 3D radiative transfer models that are used to simulate the polarized reflectance of both simplified and realistic cloud scenes. Our retrievals from actual RSP measurements performed during two field campaigns (to be published elsewhere) appear to be consistent with the correlative in situ observations within $50-100 \mathrm{~m}$ of the cloud top.

\section{RSP measurements}

The RSP (Cairns et al., 1999) is an airborne prototype for the satellite Aerosol Polarimetery Sensor (APS), which was built as part of the NASA Glory Project (Mishchenko, 2006; Mishchenko et al., 2007). This instrument measures $I, \mathrm{tQ}$ and $U$ components of the Stokes vector (cf. Hansen \& Travis, 1974; Mishchenko et al., 2006) in 9 spectral channels with center wavelengths of $410,470,555,670,865,960$,
1590,1880 and $2260 \mathrm{~nm}$. The total and polarized reflectances ( $R$ and $R_{\mathrm{p}}$ respectively) are then derived from these Stokes parameters as

$R=\frac{\pi I}{\mu_{\mathrm{s}} I_{0}}$

and

$R_{\mathrm{p}}=-\frac{\pi Q}{\mu_{\mathrm{s}} I_{0}}$

where $I_{0}$ is the extraterrestrial solar irradiance, and $\mu_{\mathrm{s}}$ is the cosine of the solar zenith angle (SZA). The Stokes parameter $Q$ in Eq. (2) is defined with respect to the scattering plane containing both solar and view directions (parameter $U$ in this plane is negligibly small). In our notation the polarized reflectance is positive when the polarization direction is parallel to the scattering plane and negative when it is orthogonal to that plane. (Note, that this notation is the opposite to that adopted by Bréon and Goloub (1998) and Bréon and Doutriaux-Boucher (2005).

The RSP scans its 14 mrad field of view over $\pm 60^{\circ}$ from nadir (starting at forward direction) and making samples at $0.8^{\circ}$ intervals, with additional calibration measurements being made in other parts of the scan. Thus, the observational data obtained in each scan consists of around 150 instantaneous Earth viewing measurements. Usually the RSP is oriented to scan along the aircraft track. In this configuration the data from the actual RSP scans can be aggregated into "virtual" scans consisting of the reflectance at the full range of viewing angles at a single point on the ground, or at the cloud top (Fig. 1). This aggregation can be done using either the aircraft attitude data (altitude, speed, pitch and crab angles), or the RSP measurements of brightness contrast between small clouds and the surface.

In this study we focus on liquid water clouds with cloud tops warmer than $0{ }^{\circ} \mathrm{C}$. For colder cloud tops, the cloud thermodynamic phase (ice, water or mixed-phase clouds) can be determined by analyzing the magnitude of the main rainbow feature (Goloub et al., 2000; van Diedenhoven et al., 2012b). Besides liquid water cloud properties RSP measurements can be used for accurate retrievals of ice cloud parameters (Ottaviani et al., 2012; van Diedenhoven et al., 2012a), aerosol optical depth, size, and refractive index (Cairns et al., 2009; Knobelspiesse et al., 2011a, 2011b; Waquet et al., 2009), as well as for characterization of the ground surface (Knobelspiesse et al., 2008). Over ocean, in addition to aerosol retrievals, chlorophyll concentrations are simultaneously estimated from the atmospherically corrected ocean color measured by the RSP (Chowdhary et al., 2005, 2006, 2012; Gilerson et al., 2006).

\section{Retrieval algorithm}

For cloud droplet size retrievals we use the scattering angle dependences of the polarized reflectance. In this study we mostly use the $865 \mathrm{~nm}$ spectral channel, however, other RSP channels can also be used (except for 960 and $1880 \mathrm{~nm}$ channels affected by water vapor and/or $\mathrm{CO}_{2}$ absorption). Our technique is focused on the sharply defined structure (rainbow) in the polarized reflectance of clouds within the scattering angle range between $137^{\circ}$ and $165^{\circ}$ (Fig. 2). The dependence of the rainbow signature on the cloud droplet effective radius has the form of a dilation of the curve along the scattering angle axis, while increase of the effective variance results in smoothing of the curve making the extrema less pronounced (until their eventual disappearance).

To analyze the shape of the polarized rainbow, we first perform a rotation from the measurement coordinate frame to that of the scattering plane (cf. Hansen \& Travis, 1974). Since the rainbow shape is determined mainly by the single scattering, this procedure results in an increase in magnitude of the Stokes parameter $Q$, while the 

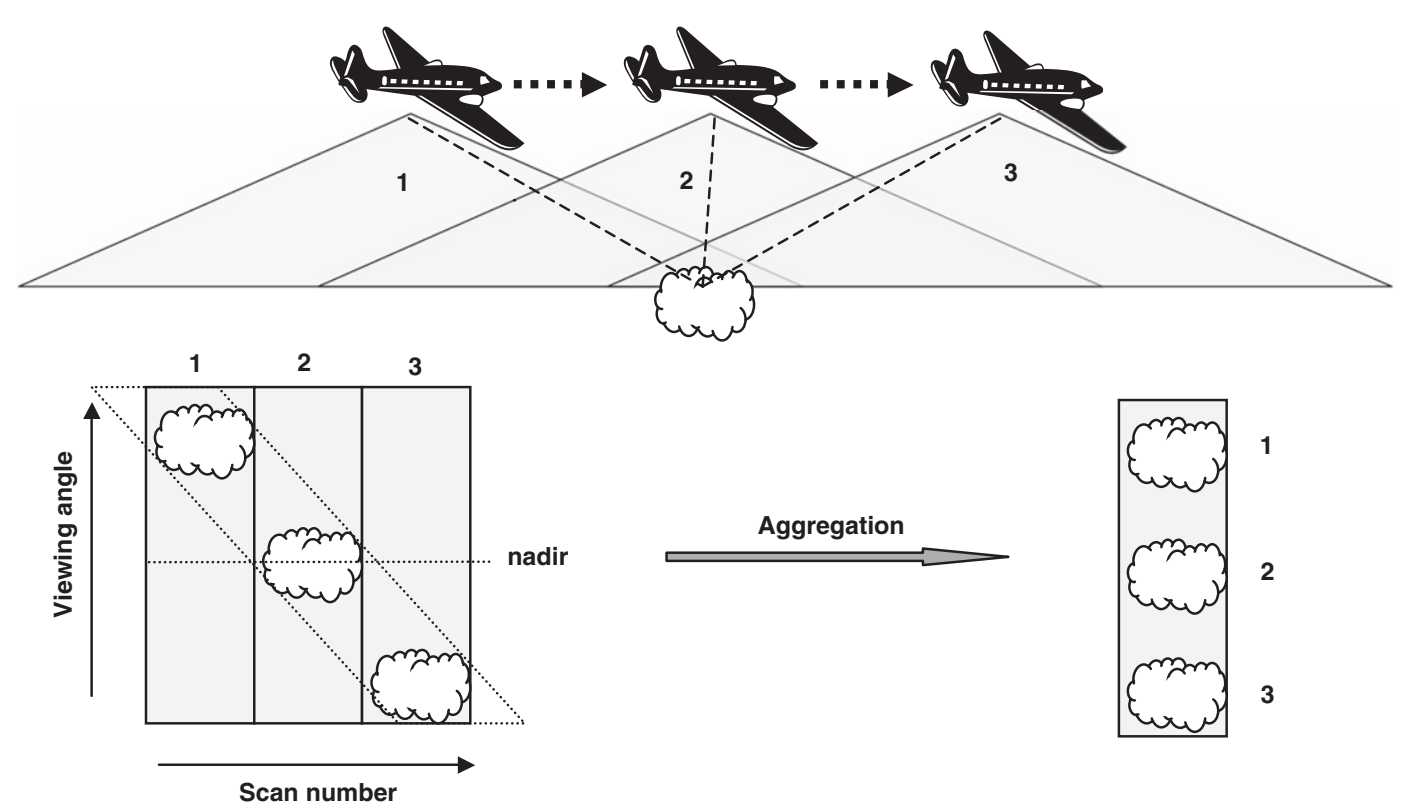

Fig. 1. Schematic illustration of the RSP data aggregation process: the data from the actual scans is aggregated into "virtual" scans, each consisting of all reflectances (at different viewing angles) from a single point at the cloud top.

parameter $U$ becomes an order of magnitude smaller than $Q$ ( $U$ would become 0 in the case of complete absence of multiple scattering). Then, we fit the polarized reflectance computed according to Eq. (2) by functions from the following parametric family:

$R_{\mathrm{p}}(\gamma)=A \cdot P_{12}^{(\mathrm{Mie})}\left(\gamma+\delta ; r_{\mathrm{eff}}, v_{\mathrm{eff}}\right)+B \cdot \cos ^{2} \gamma+C$

Here $\gamma$ is the scattering angle, $r_{\text {eff }}$ and $v_{\text {eff }}$ are respectively the effective radius and variance of the cloud droplet size distribution (cf. Hansen \& Travis, 1974), which is assumed to have the form of a gamma distribution. The phase matrix elements $P_{12}^{(\mathrm{Mie})}$ are computed using Mie theory for a grid of effective radius and variance values, while $A, B$, and $C$ are empirical fitting parameters accounting for contributions to the polarized reflectance from everything beyond single scattering by cloud droplets, such as multiple scattering, Rayleigh scattering, aerosol extinction, ground surface reflectance for thin clouds, as well as effects caused by rotation to the scattering plane. Furthermore, the effect of overlying cirrus clouds is expected to be largely mitigated by incorporating these empirical fitting parameters in the procedure ( $B$ in particular), since measurements indicate that natural ice clouds generally have featureless polarized reflectances, which are nearlinear as a functions of scattering angle (C.-Labonnote et al., 2000; Knap et al., 2005; van Diedenhoven et al., 2012b). Small fitting parameter $\delta$ is supposed to compensate for angular errors (shifts) in measured and simulated reflectances. The $\cos ^{2} \gamma$ term is included to capture any Rayleigh scattering contributions to the observations. Our tests showed that this parameterization provides a slightly better fit to simulated data than the parameterization of Bréon and Goloub (1998), which uses a term linear in $\gamma$. Note, however, that the difference between the two parameterizations is small since in the rainbow region $\cos ^{2} \gamma$ can be closely approximated by a linear function of $\gamma$ (plus a constant).

We use a pre-calculated look-up table (LUT) of $P_{12}^{(\mathrm{Mie})}$ values with $0.2^{\circ}$ resolution in the scattering angle. The grid values of $r_{\text {eff }}$ used in this LUT range from 5 to $20 \mu \mathrm{m}$ with $0.5 \mu \mathrm{m}$ increments, while the grid for $v_{\text {eff }}$ starts with the values $0.01,0.03,0.05$, and then becomes regular with 0.025 increments and maximum value of 0.35 . Our fitting technique consists of 2 steps: first, we count minima and
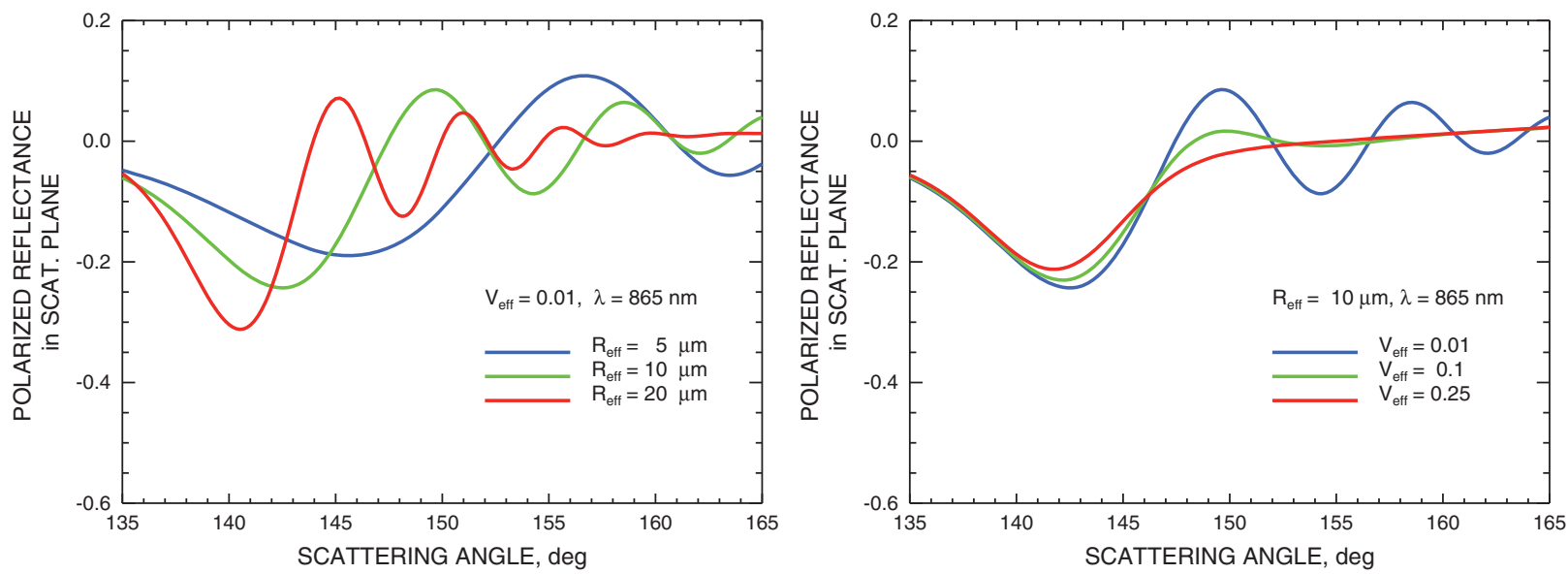

Fig. 2. Sensitivity of polarized reflectance in the rainbow angular range to effective radius (left) and variance (right) of the cloud droplet size distribution. The data are simulated for $865 \mathrm{~nm}$ wavelength in single scattering approximation. 
maxima in the observed rainbow signature and match these numbers (give or take one) with those from the corresponding LUT; after that we directly look for the best fit (according to Eq. 3) among the plausible subset of forward modeled curves selected on the first step. After the best fit cloud size model is determined we perform a refinement procedure in its neighborhood on a 10 times denser grid in effective radius and variance. The first step can be skipped for analysis of simulated data, unless noise and/or measurement artifacts are deliberately introduced into the simulations.

\section{Tests on 1D plane-parallel simulations}

To justify our (semi-empirical) method, we need to demonstrate on simulated data that the parameterization (3) adequately separates the cloud single scattering contribution from other factors providing high accuracy retrievals. We use a vector multiple scattering radiative transfer (RT) model to simulate RSP measurements of polarized reflectances emerging from a cloud field with known microphysical parameters. Then we derive cloud droplet size from these data using our retrieval algorithm, and compare the retrieved values of the droplet effective radius and variance with those assumed in the simulations.

The modified vector doubling/adding code (Cairns et al., 1997) used for forward modeling of reflectances for plane-parallel (1D) atmospheres is essentially the same as that presented by Hansen and Travis (1974) with modifications based on the work of de Haan et al. (1987). The simplicity of this RT model allowed us to perform several numerical experiments in order to study the sensitivity of the retrieval accuracy to various factors, such as the scene geometry, presence of a thick aerosol layer above the cloud, and composition of the cloud itself. We do not investigate the influence of the ground surface, since its polarized reflectance is generally smooth as a function of scattering angle and should be eliminated by the regression (3). All simulations described below were made for the $865 \mathrm{~nm}$ wavelength, unless specified otherwise.

We start with the most straight-forward numerical experiment, when the RSP data is simulated for the measurements in the principal plane (containing the Sun direction and the normal to the surface). In this case the Sun is directly ahead or behind the aircraft (so that the relative solar azimuth is either $0^{\circ}$ or $180^{\circ}$ ). The RSP measurements in this case cover the maximal scattering angle range for the given SZA. No transformation of the Stokes vector is needed, since in this situation the scattering plane corresponding to each viewing angle coincides with the principal plane. Thus, no errors are introduced in association with such a transformation. We performed this experiment on an extensive grid of realistic cloud size parameters, with $r_{\text {eff }}$ values of $5,7.5,10,12.5,15$, and $17.5 \mu \mathrm{m}$, and $v_{\text {eff }}$ values of 0.01 , $0.05,0.1$, and 0.2 . In all cases SZA was $60^{\circ}$, and the cloud optical depth (COD) was five, which is effectively infinite for the generation of polarization. We allowed the angular shift fitting parameter $\delta$ to vary within $\pm 0.2^{\circ}$ (which is the resolution of both the forward model and the LUT used for inversions) with $0.01^{\circ}$ increments. The fitting errors are computed as the standard deviations of the differences between the simulated and fitting values on the angular grid. Examples of the simulated and best fit (according to Eq. (3)) polarized reflectance are shown in Fig. 3 by respectively red and green curves for $r_{\text {eff }}=7.5,17.5 \mu \mathrm{m}$, and $v_{\text {eff }}=0.01,0.2$. The blue curves show the reflectance computed assuming exactly the same size distribution parameters as those used in the RT model. They are slightly different from the best-fit curves, partly due to the small angular uncertainties (best seen in the top right panel). The comparison between the retrieved effective radius and variance values with those assumed in the simulations is shown in Fig. 4 as separate scatter plots for $r_{\text {eff }}$ and $v_{\text {eff }}$ and also as the difference vectors in $\left(r_{\text {eff, }}, v_{\text {eff }}\right)$-plane. There the diamonds represent the assumed models, and the triangles are the best-fit retrievals. We see from Fig. 4 (top) that the mean accuracy of the $r_{\text {eff }}$ retrievals is better than $0.1 \mu \mathrm{m}$, while its standard deviation has maximal value of $0.21 \mu \mathrm{m}$ at $v_{\text {eff }}=0.2$ (due to the single worst case shown in Fig. 3 (top right)). While the error in the effective radius retrievals is rather random, the derived values of $v_{\text {eff }}$ show systematic biases (Fig. 4 (middle)), which can be approximated by $r_{\text {eff-dependent }}$ linear functions of the assumed $v_{\text {eff }}$ (they are listed at the bottom of the plot). We see that these biases range from $6 \%$ to $27 \%$ and generally decrease with $r_{\text {eff, }}$ while the largest absolute differences occur at large

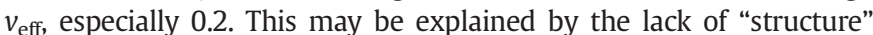
(number and amplitude of oscillations) in the angular dependence of polarized reflectance for both small sizes and large variances, as can be seen in Fig. 2. The "smoothing" effect of multiple scattering on the polarized reflectance, is then interpreted by the retrieval algorithm as a bias toward larger $v_{\text {eff }}$, and (to a much lesser extent) to smaller droplet size.

Similar small biases can be observed in droplet sizes retrieved from different spectral channels. Fig. 5 shows polarized reflectances and size retrievals for the same models as presented in the top panels of Fig. 3 ( $\left.r_{\text {eff }}=7.5 \mu \mathrm{m}, v_{\text {eff }}=0.01,0.2\right)$, but for a shorter $(410 \mathrm{~nm})$ and a longer (1588 nm) wavelength. The increasing lack of "structure" in these curves causes some underestimation (by 0.1-0.6 $\mu \mathrm{m}$ ) of the droplet $r_{\text {eff }}$ as wavelength increases, and the larger retrieval errors are observed for large $v_{\text {eff. }}$. While these biases are relatively small for simulated data, the presence of measurement noise or artifacts is likely to more strongly affect the retrievals when the rainbow signature is weak. Thus, we do not recommend using the longest RSP wavelengths (1588 and $2260 \mathrm{~nm}$ ) alone. Our tests show that polarized reflectance in a single channel is sufficient for accurate determination of droplet size distribution parameters. However, simultaneous retrievals using multiple spectral channels are beneficial for providing a retrieval consistency check, as well as a quality assessment of the measurements (particularly for airborne measurements where pointing knowledge is unlikely to be better than $\pm 1^{\circ}$ ).

\section{Effects of rotation to the scattering plane}

Before describing the effect of the coordinate frame rotation on the droplet size retrievals, we should note that the range of the observed scattering angles also depends on solar/viewing geometry, and this is an important factor affecting the retrieval accuracy. Obviously, good retrievals cannot be expected, when only a small part of the rainbow structure is seen in the data. Fig. 6 shows the dependences of the scattering angle range on the relative azimuth between the Sun and aircraft directions for three different solar zenith angles $\left(40^{\circ}, 60^{\circ}\right.$, and $\left.80^{\circ}\right)$, assuming that the viewing angle varies within $\pm 60^{\circ}$ from nadir. The relative azimuth intervals are classified in these plots according to the extent ("Full", "Partial", or "None") to which the rainbow region (here $140^{\circ}-165^{\circ}$ ) is covered by the RSP measurements made under the corresponding viewing geometries. These results are summarized in the diagram in Fig. 6 (bottom right). We see from this diagram that in the limit case, when the Sun is directly at zenith, the full rainbow range is observed independently of the relative Sun/aircraft azimuth. For non-zero SZA the best rainbow coverage is achieved in the principle plane (zero relative azimuth), then, the coverage decreases with the azimuth as it increases to $90^{\circ}$ (values larger than $90^{\circ}$ are not shown due to symmetry). The coverage also gets worse with increase in SZA, so that for SZA $>75^{\circ}$ full-range rainbow observation cannot be made even in the principal plane while at $\mathrm{SZA}>40^{\circ}$ there is a relative azimuth range, where the rainbow cannot be seen at all.

As we already mentioned, the polarized reflectance measured in the solar principal plane is determined by the Stokes parameter $Q$ according to Eq. (2), while the parameter $U$ is zero as a result of symmetry. In order to perform the droplet size retrievals in an arbitrary viewing geometry the Stokes vector needs to be transformed from the measurement coordinate frame into that of the scattering plane. Note, that this plane is defined as the one containing both solar and viewing directions, thus, in a generic case it is different for different 

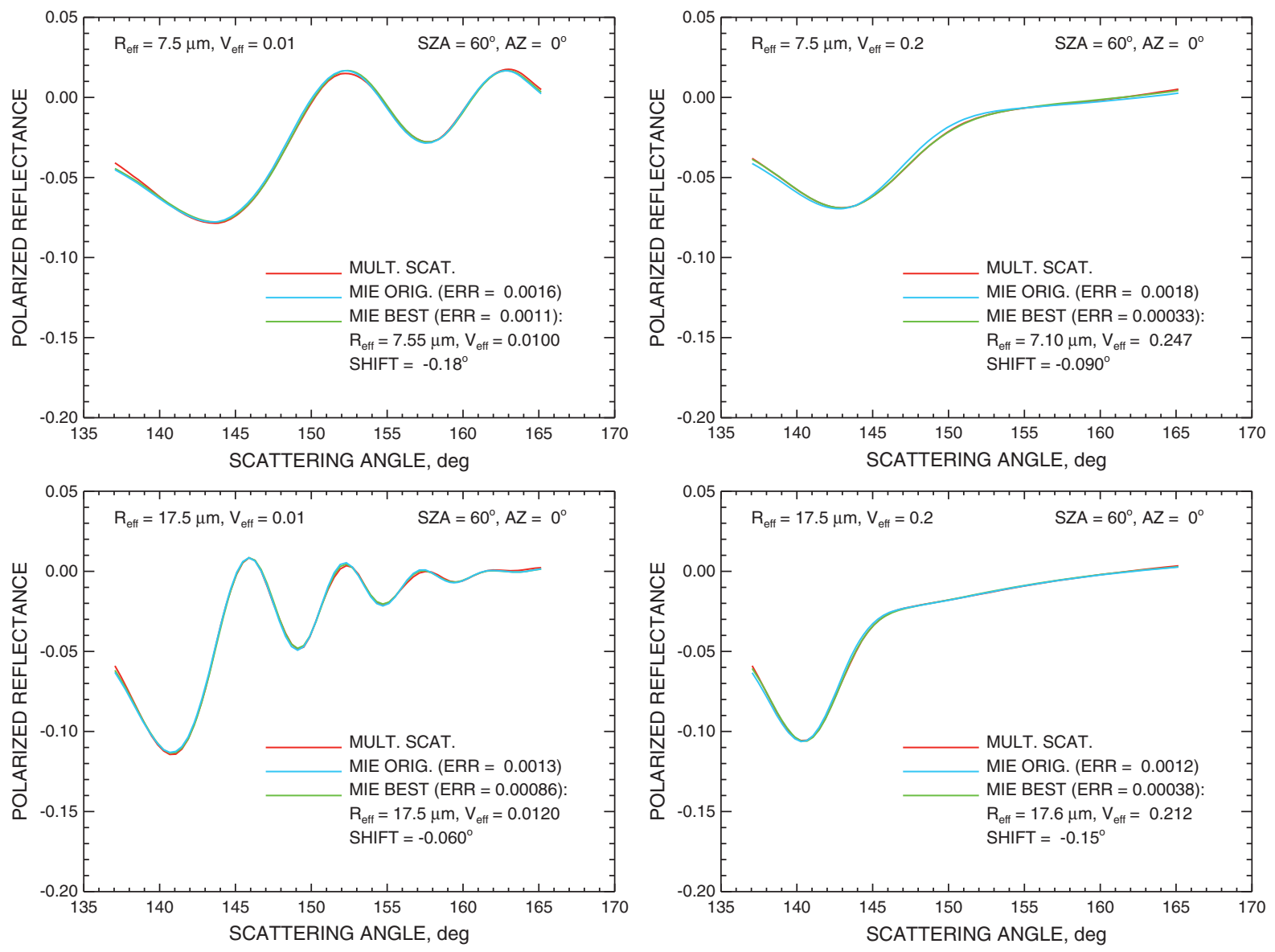

Fig. 3. Retrievals of effective radius and variance from the polarized reflectances simulated using plane-parallel adding/doubling vector code for $r_{\text {eff }}=7.5$ and $17.5 \mu$, and $v_{\text {eff }}=0.01$ and 0.2 . The simulated (using multiple scattering) reflectances are shown in red; the single-scattering fits assuming the same $r_{\text {eff }}$ and $v_{\text {eff }}$ that were used in the radiative transfer model are in blue; while the best fits (assuming an angular shifts) are in shown in green. In all cases COD is 5.

viewing angles within the same RSP scan. In the case of single scattering, the transformation of the Stokes vector corresponding to the rotation of the reference frame is linear (cf. Hansen \& Travis, 1974) and affects only its $Q$ and $U$ components ( $U=0$ after this rotation). While, strictly speaking, such a simple transformation is not valid in the case of multiple scattering in clouds it can still be used in the rainbow region, where the angular structure of polarized reflectance is dominated by single scattering. In this case the transformed $U$ is orders of magnitude smaller than the transformed $Q$ which has similar structure in the rainbow to that of single scattering. The deviation in the parameter $Q$ from that of single scattering, resulting from the reference frame rotation under multiple scattering conditions, is expected to be a smooth function of scattering angle, which is absorbed by the last two terms in the parameterization (3). This assumption is confirmed by the excellent fits of the rotated polarized reflectances using Eq. (3) in the numerical tests described below.

We applied our retrieval algorithm to RSP measurements simulated for 3 solar zenith angles $\left(20^{\circ}, 40^{\circ}\right.$, and $\left.60^{\circ}\right)$ and the corresponding relative azimuths $\left(48^{\circ}, 22^{\circ}\right.$, and $\left.16^{\circ}\right)$ chosen to be the most distant from $0^{\circ}$, while still allowing for full rainbow range observation (see Fig. 6). This choice was made to separate the errors introduced by the frame rotation from the loss of accuracy due to reduced angular range. For comparison we also generated and analyzed data for the same SZAs, but with the measurements made in the principal plane. The cloud parameters assumed in simulations were the same in all cases: $r_{\text {eff }}=17.5 \mu \mathrm{m}$ and $v_{\text {eff }}=0.1$, and COD $=5$. The polarized reflectances obtained after the transformation into scattering plane frame are shown in Fig. 7. We see that the "rainbow signal" is weaker at smaller SZA, introducing larger uncertainties in the retrievals, especially for $\mathrm{SZA}=20^{\circ}$ (which, however are still quite good: only $0.5 \mu \mathrm{m}$ underestimation of $r_{\text {eff, }}$ and $10 \%$ overestimation of $\left.v_{\text {eff }}\right)$. The "U-reflectances" computed using the analog of Eq. (2), but with $Q$ replaced by the residual value of $U$ in the scattering plane are shown in Fig. 7 (bottom right). One can see that these residues are more than 2 orders of magnitude smaller than the corresponding polarized reflectances (while larger than the corresponding quantities in the principle plane simulations, which are essentially 0 (smaller than $10^{-9}$ )). The differences between retrievals from the "rotated" datasets and from their principle plane counterparts $\left(r_{\text {eff }}=17.2 \mu \mathrm{m}, v_{\text {eff }}=0.110\right.$ for $\mathrm{SZA}=20^{\circ}, r_{\mathrm{eff}}=17.5 \mu \mathrm{m}, v_{\mathrm{eff}}=0.107$ for $\mathrm{SZA}=40^{\circ}$, and $r_{\mathrm{eff}}=$ $17.5 \mu \mathrm{m}, v_{\text {eff }}=0.102$ for $\mathrm{SZA}=60^{\circ}$ ) are as little as $0.1-0.2 \mu \mathrm{m}$ in radius and a few percentage points in variance. This allows us to conclude that the error introduced by the transformation of the Stokes vector to the scattering plane frame and omission of $U$ is negligible.

\section{Effects of angular shifts}

Angular errors, if unaccounted for, can significantly affect the accuracy of satellite, and especially airborne measurements. In particular, inadequately recorded aircraft pitch, roll and yaw may cause an error in the computed scattering angle. In realistic situation this error is a non-linear function of the aircraft attitude uncertainties, which can be large. However, if these uncertainties are small (less than a degree), they result in an effective shift in scattering angle, which is easy to model. To see the effect of such a shift on the droplet size retrievals, we took the polarized reflectance simulated for clouds with $r_{\text {eff }}=7.5$ 

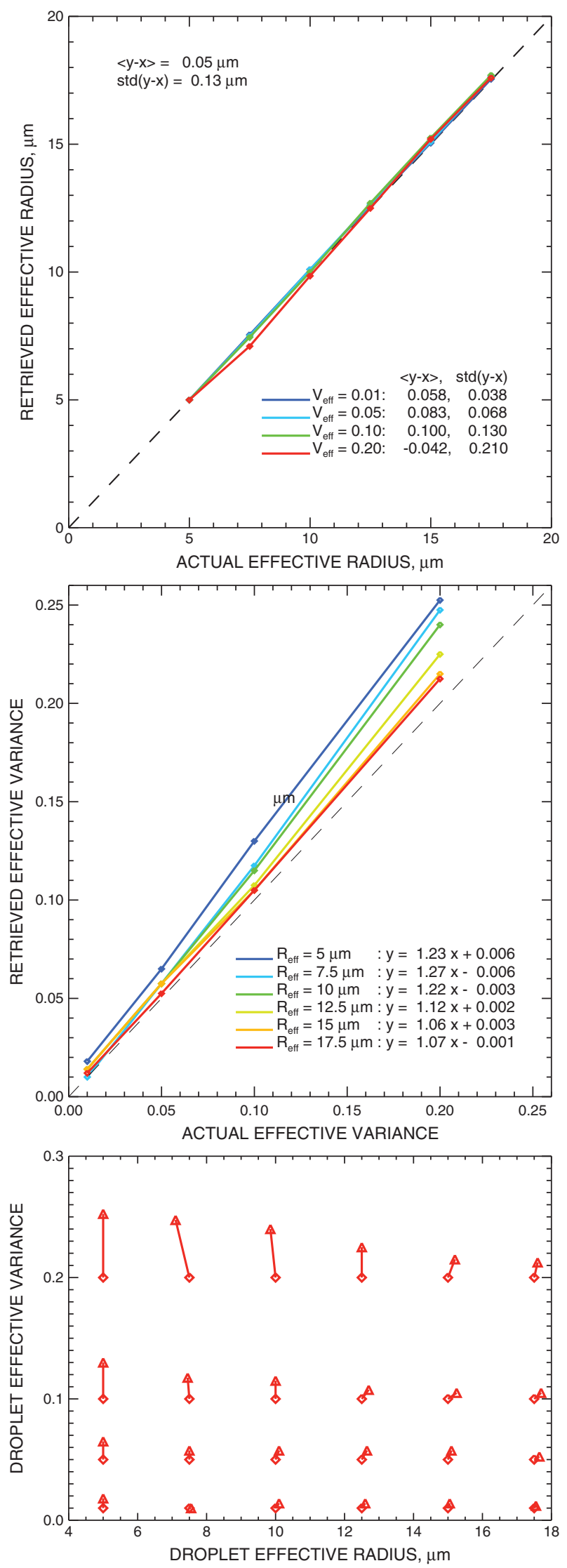

Fig. 4. Comparison of retrievals of effective radius (top) and variance (middle) with the actual values assumed in plane-parallel vector simulations (in all cases COD $=5$ ). Bottom: the same comparison in $\left(r_{\text {eff }}, v_{\text {eff }}\right)$-plane. Diamonds depict the actual values assumed in simulation, triangles - best fit retrievals. and $17.5 \mu \mathrm{m}$ and displaced the actual scattering angle records by small angle $\Delta \gamma$ ranging from $-0.5^{\circ}$ to $0.5^{\circ}$. In all considered cases $v_{\text {eff }}=0.1, \mathrm{COD}=5, \mathrm{SZA}$ is $60^{\circ}$, and the relative solar-aircraft azimuth is $0^{\circ}$. The results of application of our algorithm (without optimization for angular shift parameter $\delta$ ) to this dataset are presented in Fig. 8 for the case of $r_{\text {eff }}=17.5$ (plots in the case of $r_{\text {eff }}=7.5$ are similar). A strong linear dependence of the retrieved $r_{\text {eff }}$ on $\Delta \gamma$ is seen in the top right panel of Fig. 8. This seems reasonable when one considers that, while the dependence of the rainbow shape on the droplet effective radius can be characterized as a dilation with fixed point at $135^{\circ}$ in scattering angle space (as $r_{\text {eff }}$ decreases), small shifts can also be interpreted as dilations. To see this, we can apply shift $\Delta \gamma$ to both ends of the scattering angle interval $\left[\gamma_{0}, \gamma_{1}\right]$. At each end the shift can be interpreted as a multiplication by a factor $c_{i}=$ $1+\left(\Delta \gamma / \gamma_{i}\right), i=0.1$. The factors $c_{0}$ and $c_{1}$ are identical in the case of true dilation, while in our case they are very close as $\Delta \gamma \ll \gamma_{0}<\gamma_{1}$.

The accuracy of the numerical simulations used for these tests was $0.2^{\circ}$, so it is not surprising to see a discrepancy of that order between simulated multiple- and single-scattering polarized reflectances. In the case of $r_{\text {eff }}=7.5 \mu \mathrm{m}$ the size assumed in the model can be retrieved when applying a $-0.25^{\circ}$ shift, while the effective radius value retrieved without a shift was $7.2 \mu \mathrm{m}$. Given the linearity of the dependence of the retrieved $r_{\text {eff }}$ on the implied $\Delta \gamma$, this means that the retrieval error in this case is $1.2 \mu \mathrm{m}$ or $16 \%$ per degree of the shift. In the case of $r_{\text {eff }}=17.5 \mu \mathrm{m}$ (Fig. 8) a smaller $-0.15^{\circ}$ shift was necessary to recover the assumed droplet size, while $r_{\text {eff }}=$ $16.8 \mu \mathrm{m}$ was retrieved with $\Delta \gamma=0$. This corresponds to $4.7 \mu \mathrm{m}$ or $27 \%$ error rate per degree of the shift. The error associated with angular uncertainty is more significant for larger particles, since the oscillating structure of the rainbow generated by them has shorter angular period than when the droplets are smaller (see Fig. 2). Thus, the same angular shift appears to be larger relative to this period for larger droplets, and, therefore, has stronger effect on retrievals. The effect of the shift on the retrieved $v_{\text {eff }}$ is weaker, and practically negligible in the case of $r_{\text {eff }}=17.5 \mu \mathrm{m}$ (Fig. 8 (middle right)).

The bottom right panel of Fig. 8 demonstrates that the uncertainties in scattering angle shift can be effectively resolved if the magnitude of the shift is taken as a free parameter in the fitting procedure, since the best fits to the simulated reflectances occur with the same $\Delta \gamma$, for which the retrieved values of $r_{\text {eff }}$ coincide with those assumed in the model. This feature is implemented in our algorithm by introduction of the shift parameter $\delta$ in Eq. (3), which is allowed to vary within $\pm 0.2^{\circ}$ for simulated data tests. In the case of real airborne data analysis, when the measurements can be affected by uncertainties in aircraft attitude, we first correct the pitch angle, so that the first (and the deepest) minimum in polarized reflectance is between $140^{\circ}$ and $145^{\circ}$, and then apply fitting procedure, which includes optimization with respect to smaller angular errors.

\section{Aerosol layer over cloud}

We performed a number of simulations of RSP measurements over a mixed atmosphere consisting of a cloud and an aerosol layer on top of it. This allowed us to study the effect of aerosols on cloud droplet size retrievals, and also to evaluate the RSP algorithm's sensitivity to a rainbow signal originated deep in the scattering medium. All simulations were performed for the same viewing geometry (principal plane, $\left.\mathrm{SZA}=60^{\circ}\right)$ and cloud parameters $\left(r_{\mathrm{eff}}=17.5 \mu \mathrm{m}, v_{\mathrm{eff}}=0.1\right.$, and COD $=5$ ). The aerosol layer was modeled by small water particles $\left(n_{r}=1.3275\right.$ (same as in cloud), $r_{\text {eff }}=0.15 \mu \mathrm{m}$ and $\left.v_{\text {eff }}=0.1\right)$, and had varying optical depth $(\mathrm{AOD}=0,0.1,0.25,0.5,0.75,1$, and 2$)$. The polarized reflectance of the aerosol alone increases monotonically with the scattering angle between $137^{\circ}$ and $165^{\circ}$, and does not exhibit any rainbow features. Addition of the aerosol layer on top of the cloud leads to a decrease in the "rainbow signal" magnitude, that affects the droplet size retrieval accuracy. The dependence of the 

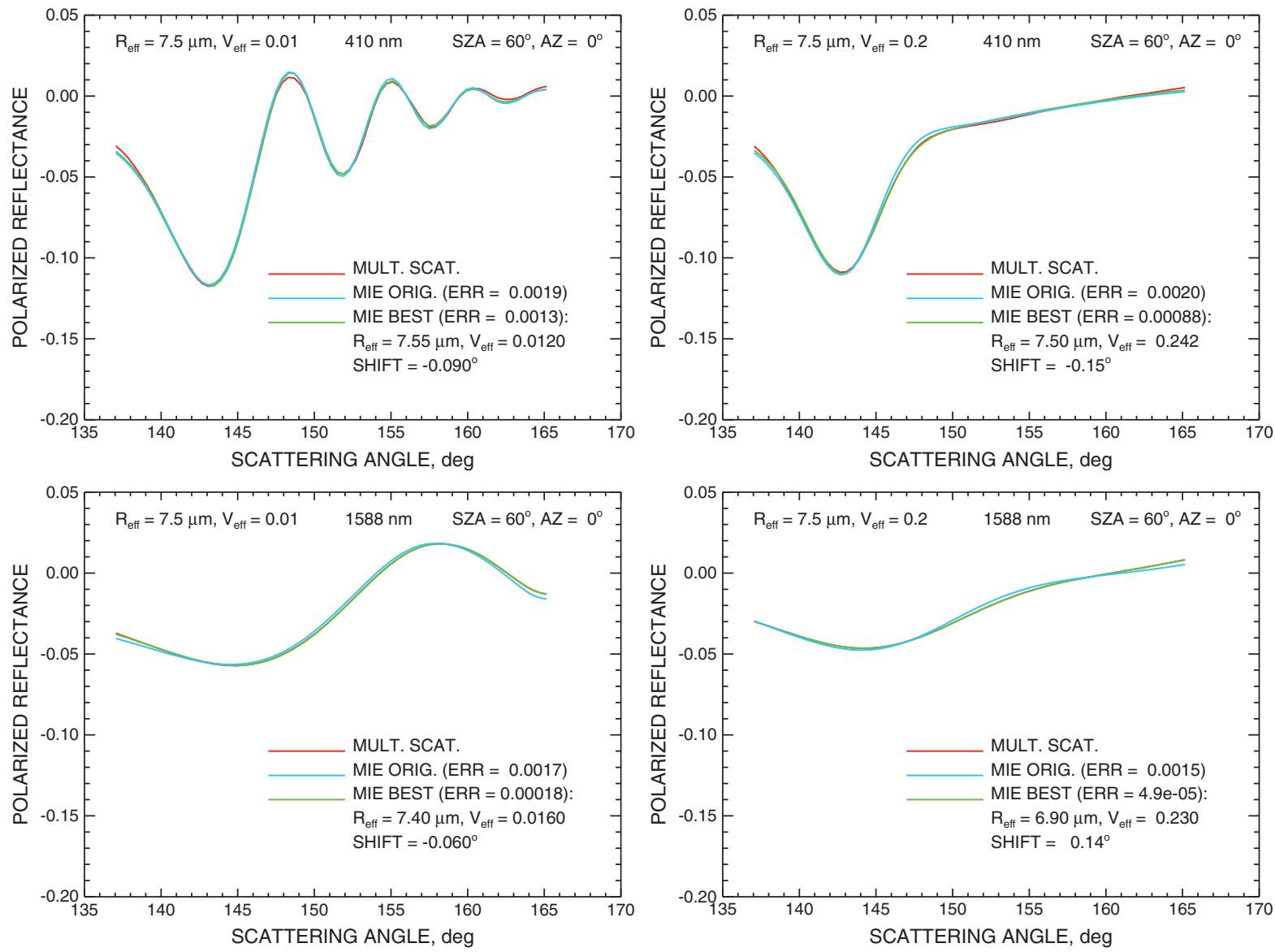

Fig. 5. Same as Fig. 3 (top) but for $410 \mathrm{~nm}$ (top) and $1588 \mathrm{~nm}$ (bottom) wavelengths.

retrieved droplet effective radius and variance on the thickness of the aerosol layer is weak, and the retrieval errors are similar to the cloud-only case: less than $0.5 \mu \mathrm{m}$ in $r_{\text {eff }}$ and $0.04 v_{\text {eff. }}$. The largest errors occur at the largest AOD value (0.75), for which retrievals were still possible despite an increasing lack of "structure" in the dataset.

Fig. 9 illustrates a simple test characterizing the effect of the aerosol layer on the rainbow signal magnitude, based on the simulations described above. We took all polarized reflectances simulated for different AOD (top left panel in Fig. 9) and represented them in the form similar to Eq. (3)

$R_{\mathrm{p}}\left(\gamma ; \tau_{\mathrm{a}}\right)=A^{\prime} R_{\mathrm{p}}\left(\gamma ; \tau_{\mathrm{a}}=0\right)+B^{\prime} \gamma+C^{\prime}$

where $\tau_{\mathrm{a}}$ is AOD, and $A^{\prime}=A^{\prime}\left(\tau_{\mathrm{a}}\right)$ is the magnitude of the rainbow signal relative to the case with no aerosol presence $\left(\tau_{\mathrm{a}}=0\right)$. Unlike Eq. (3), this form does not imply droplet size retrievals or modeling using Mie theory (we use prime accents for the coefficients to emphasize this difference). The model fit (4) is good and the residues $R_{\mathrm{p}}\left(\gamma ; \tau_{\mathrm{a}}\right)-A^{\prime} R_{\mathrm{p}}(\gamma ; 0)$ can be very closely fitted by linear functions of the scattering angle $\gamma$ (Fig. 9(top right)). These residuals can be attributed to the polarized reflectance of the aerosol layer. The dependence of the signal magnitude $A^{\prime}$ on AOD appears to be exponential (see Fig. 9 (bottom left)), and agrees well with the Beer's law attenuation model:

$A^{\prime}\left(\tau_{\mathrm{a}}\right)=\exp \left[-\left(\frac{1}{\mu_{\mathrm{s}}}+\frac{1}{\mu_{\mathrm{v}}}\right) \tau_{\mathrm{a}}\right]$.

Here $\mu_{\mathrm{s}}$ and $\mu_{\mathrm{v}}$ are cosines of respectively the solar zenith angle $\theta_{\mathrm{s}}$ and the viewing angle $\theta_{\mathrm{v}}$. Knowing the solar zenith angle $\left(\theta_{\mathrm{s}}=60^{\circ}\right)$ used in our simulations, we can estimate (see Fig. 9 (bottom right)) the value of the average viewing angle $\left(\theta_{\mathrm{v}}=29^{\circ}\right)$ from Eq. (5), and using that

$\gamma=180^{\circ}-\theta_{\mathrm{s}}+\theta_{\mathrm{v}}$

find the corresponding scattering angle $\gamma=149^{\circ}$. As one would expect, this value is in the middle of the rainbow angular range. Thus, our numerical experiment shows that the magnitude of the rainbow, in polarized reflectance observations, is attenuated by overlying aerosols according to the Beer's law but its structure is not changed by the overlying aerosol scattering.

\section{Contribution of forward scattering to the rainbow}

Our tests described in this section show that the polarized rainbow amplitude includes contributions of both single and forward-directed multiple scattering. The latter, does not affect the angular structure of the rainbow, and, therefore, droplet size retrievals. However, assessment of the magnitudes of various contributions will help to understand the retrievals from stratified clouds described in detail in the next section.

Let us determine the general functional form that the reflectance $R$ of a single-layer cloud of optical depth $\tau$ should take in the single- or forward-scattering case. $R$ must satisfy the following property, when an additional layer of $\mathrm{COD}=\tau_{1}$ is attached to the top of the cloud of $\mathrm{COD}=\tau_{2}$ :

$R\left(\tau_{1}+\tau_{2}\right)=R\left(\tau_{1}\right)+u\left(\tau_{1}\right) R\left(\tau_{2}\right)$.

Here $u(\tau)=\exp (-\beta \tau)$ is the attenuation factor. The exponent $\beta$ depends on the scattering geometry and may also be influenced by 

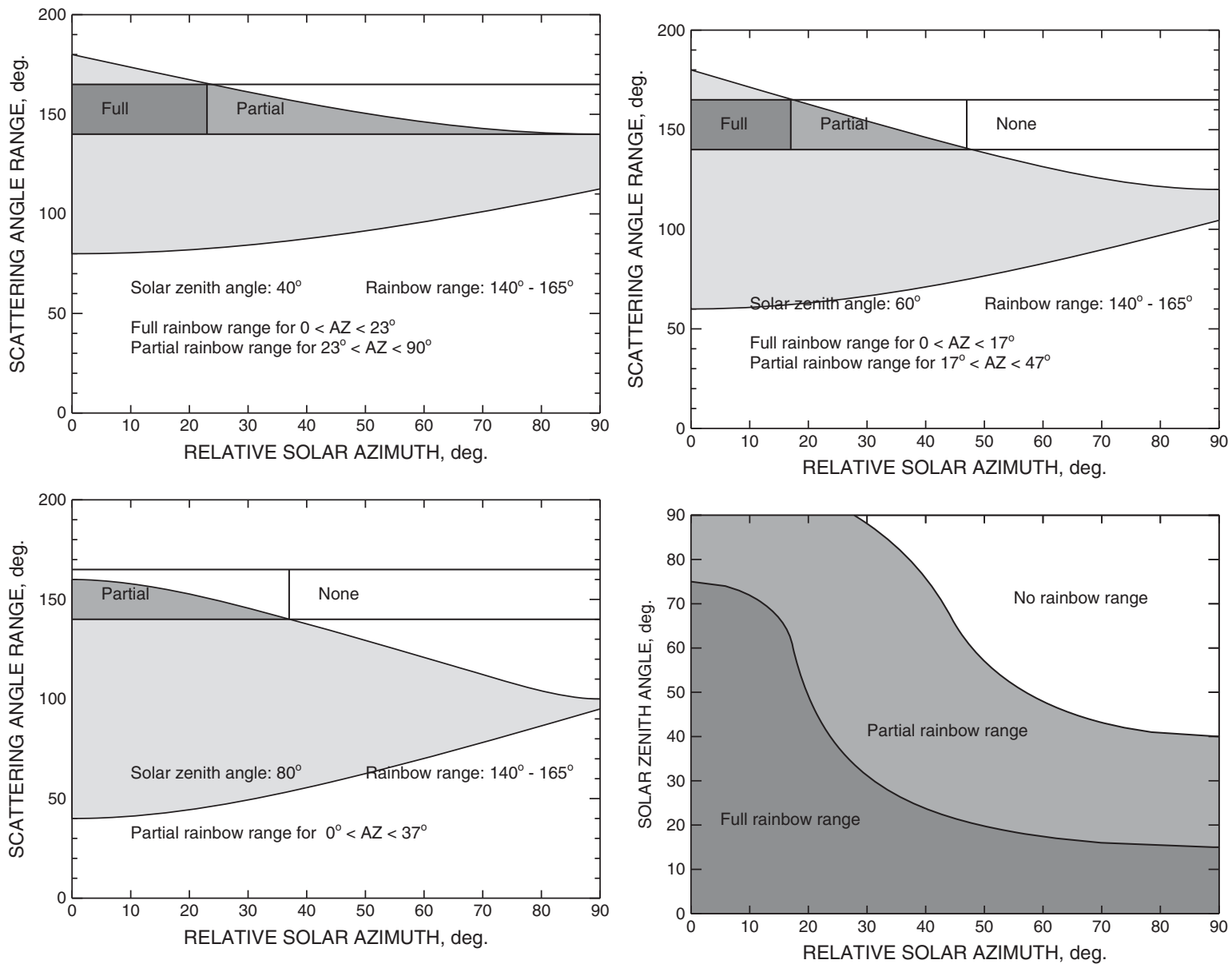

Fig. 6. Top, and bottom left: dependences of the scattering angle range viewed by RSP (light gray) on the solar azimuth (relative to the aircraft orientation) for three values of the solar zenith angle. The angular ranges where rainbow can be observed (fully or partially) are shown by darker shades of gray. Bottom right: diagram showing availability of observations in the rainbow angular range $\left(140^{\circ}-165^{\circ}\right)$ depending on the solar zenith angle and the relative solar azimuth.

multiple scattering in the forward direction, which depends on the droplet size. If we look at a rather narrow scattering range (as in the rainbow case) the explicit dependence of the attenuation factor $u$ on the scattering angle can be neglected, and we can assume that the reflectance $R$ can be factorized into a product of the $\tau$-independent angular spectrum and an attenuation function that depends on the optical depth only. Accepting these assumptions, it is not difficult to show that the only functional form of $R(\tau)$ satisfying the group transformation property (efeq: $R-t 1+t 2$ ) is

$R(\tau)=\left[1-e^{-\beta \tau}\right] R^{(\infty)}$,

where $R^{(\infty)}$ is the reflectance of semi-infinite medium with the same microphysical properties.

To test this formula, we simulated the polarized reflectance for clouds with droplet $r_{\text {eff }}=5,7.5,12.5$ and $17.5 \mu \mathrm{m}$ (in all cases $v_{\text {eff }}=$ $0.1, \mathrm{SZA}=60^{\circ}$, measurements are made in the principal plane). COD in these tests ranged between 0.1 and 0.5 with 0.1 increments. $R^{(\infty)}$ was taken from simulations with $C O D=5$, which is essentially infinite for the generation of polarization. In all cases Eq. (8) was satisfied with very high accuracy. This is demonstrated in Fig. 10 for the case of $r_{\text {eff }}=17.5 \mu \mathrm{m}$. The polarized reflectances generated for different COD values were fitted by scaled $R^{(\infty)}$ using procedure similar to Eq. (4). The corresponding scaling factors $v(\tau)$ are then used to compute the attenuation functions $u(\tau)=1-v(\tau)$, which appeared to have an exponential form. The values of attenuation exponents corresponding to $r_{\text {rmeff }}=5,7.5,12.5$ and $17.5 \mu \mathrm{m}$ are respectively $\beta=1.91,1.89,1.79$, and 1.57. All these numbers are smaller than the single-scattering airmass $\beta=3.14$, indicating that the polarized rainbow amplitude includes contribution from forward scattering within the phase function diffraction peak. This is also supported by the fact that the observed $\beta$ is smaller for larger particles, that have stronger forward scattering. At the particle size close to that of aerosol $(0.15 \mu \mathrm{m}), \beta$ is back to the single-scattering value (as it was shown in the previous section).

\section{Stratified cloud}

The size distribution of droplets in real clouds usually varies with the altitude. This raises the question of how deep can RSP "see" into a cloud and what its measurements represent regarding the statistics of the cloud droplet size profile. The assumption, that the polarized rainbow shape is dominated by single scattering, allows us to better understand the relationship between the droplet size distribution parameters retrieved from polarized reflectance at the cloud top and the profiles of these parameters inside the cloud.

\subsection{General properties}

Let us consider a vertically stratified cloud of the optical depth $\tau_{\max }$, and parameterize the altitude dependence of droplet size distribution in it by the optical depth $\tau$ measured from the top of the cloud downwards $\left(0 \leq \tau \leq \tau_{\max }\right)$. Suppose that we successfully extracted the single-scattering component $P_{12}^{(\text {top })}(\gamma)$ of the polarized reflectance measured by RSP at the cloud top. We assume that the cloud particles contribute to $P_{12}^{(\text {top })}(\gamma)$ independently one from another, thus, it is a 

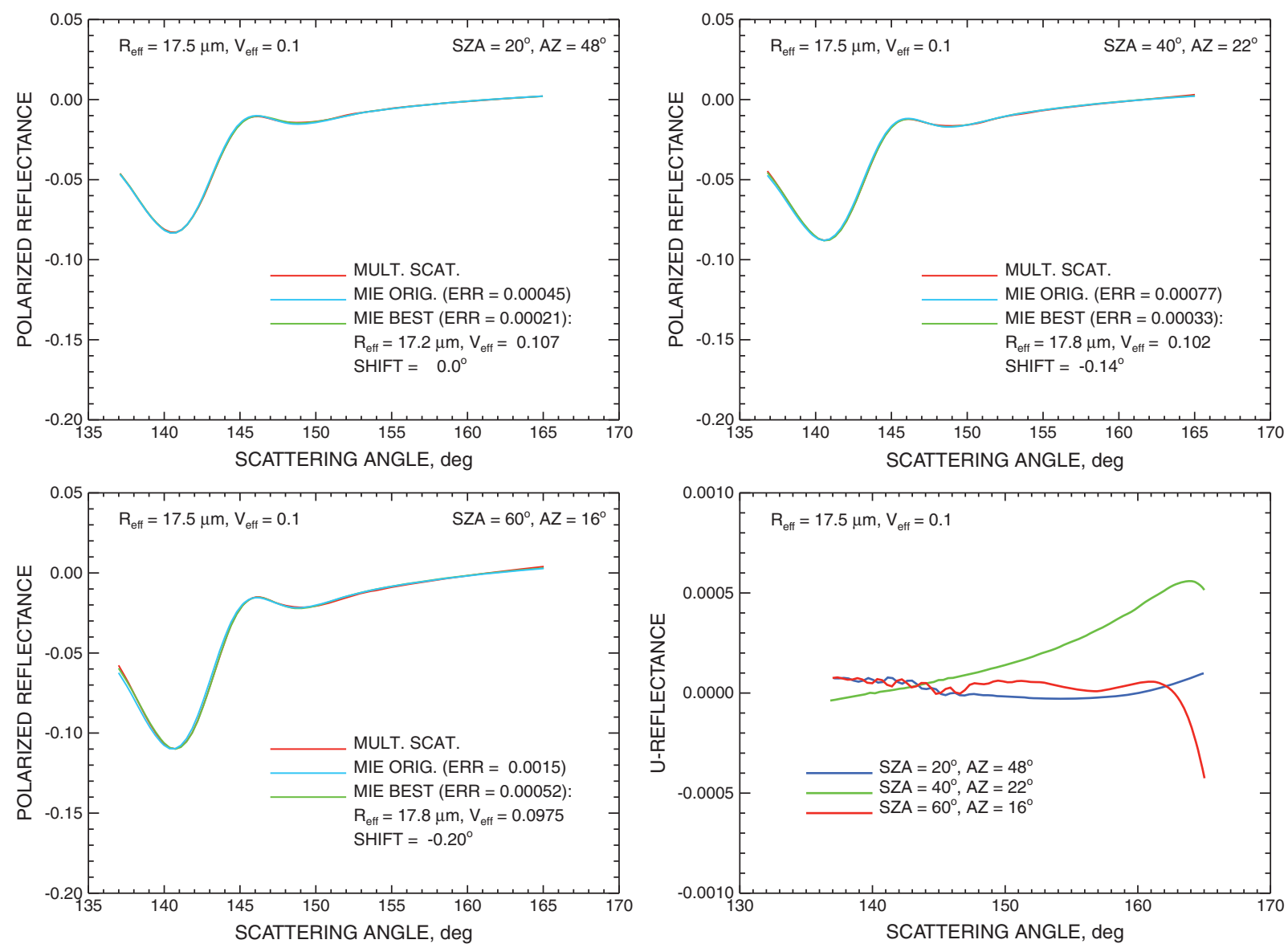

Fig. 7. Top, and bottom left: Polarized reflectances (after transformation to the scattering plane) and the corresponding retrievals from RSP measurements. The data are simulated for different Sun/viewing geometries: SZA of $20^{\circ}, 40^{\circ}$, and $60^{\circ}$, and the maximal relative solar-aircraft azimuth still allowing for full rainbow range observation at given SZA. In all cases $r_{\text {eff }}=17.5 \mu \mathrm{m}$ and $v_{\text {eff }}=0.1$, and $\mathrm{COD}=5$. Bottom right: the residual " $U$-reflectances" left after transformation to the scattering plane in these simulations.

superposition of the signals coming from different depths. This allows us to introduce the "effective" droplet size distribution $n_{\text {top }}(r)$. This distribution is derived from the RSP measurements, since it determines the polarized reflectance at the cloud top:

$P_{12}^{(\text {top })}(\gamma)=\frac{\int_{0}^{\infty} \sigma_{\text {sca }}(r) P_{12}(\gamma, r) n_{\text {top }}(r) \mathrm{d} r}{\int_{0}^{\infty} \sigma_{\text {sca }}(r) n_{\text {top }}(r) \mathrm{d} r}$,

where $\sigma_{\text {sca }}(r)$ is the scattering cross-section. The cloud-top distribution $n_{\text {top }}(r)$ is normalized by the condition

$\int_{0}^{\infty} n_{\text {top }}(r) \mathrm{d} r=1$

and it can be represented as a superposition of size distributions at different optical depths:

$n_{\text {top }}(r)=\int_{0}^{\tau_{\max }} w(\tau) n_{\tau}(r) \mathrm{d} \tau$

where $w(\tau)$ is the weighting function normalized as

$\int_{0}^{\tau_{\max }} w(\tau) \mathrm{d} \tau=1$ and $n_{\tau}(r)$ is the droplet size distribution in the optical depth interval $[\tau, \tau+\mathrm{d} \tau]$. If the latter distribution is assumed to be normalized by the condition

$\int_{0}^{\infty} n_{\tau}(r) \mathrm{d} r=1$

its weight $w(\tau)$ in $n_{\text {top }}(r)$ is determined by two factors: the number of particles $\mathrm{d} N_{c}$ (per unit area) in the corresponding optical depth interval $\mathrm{d} \tau$, and the Beer's law attenuation factor $u(\tau)$ :

$w(\tau)=C u(\tau) \frac{\mathrm{d} N_{\mathrm{c}}}{\mathrm{d} \tau}$

Here $C$ is the normalization constant derived from Eq. (12), and

$u(\tau)=\exp [-\beta \tau]=\exp \left[-\left(\frac{1}{\mu_{s}}+\frac{1}{\mu_{v}}\right) \alpha \tau\right]$

where $\alpha$ provides a correction for the effects of multiple scattering in the forward direction. The extinction optical depth of a homogeneous layer is

$\tau=N_{\mathrm{c}}\left\langle\sigma_{\text {ext }}\right\rangle=N_{\mathrm{c}}\left\langle\pi r^{2} Q_{\text {ext }}(r)\right\rangle=N_{\mathrm{c}} \pi\left\langle Q_{\text {ext }}\right\rangle\left\langle r^{2}\right\rangle$

with $N_{\mathrm{c}}$ being the $2 \mathrm{D}$ column particle concentration (i.e. the number of particles in a column over a unit area), $\sigma_{\text {ext }}$ and $Q_{\text {ext }}$ are respectively the extinction cross-section and efficiency in the layer, and the averaging is over a statistical ensemble of particle sizes specified by the size 

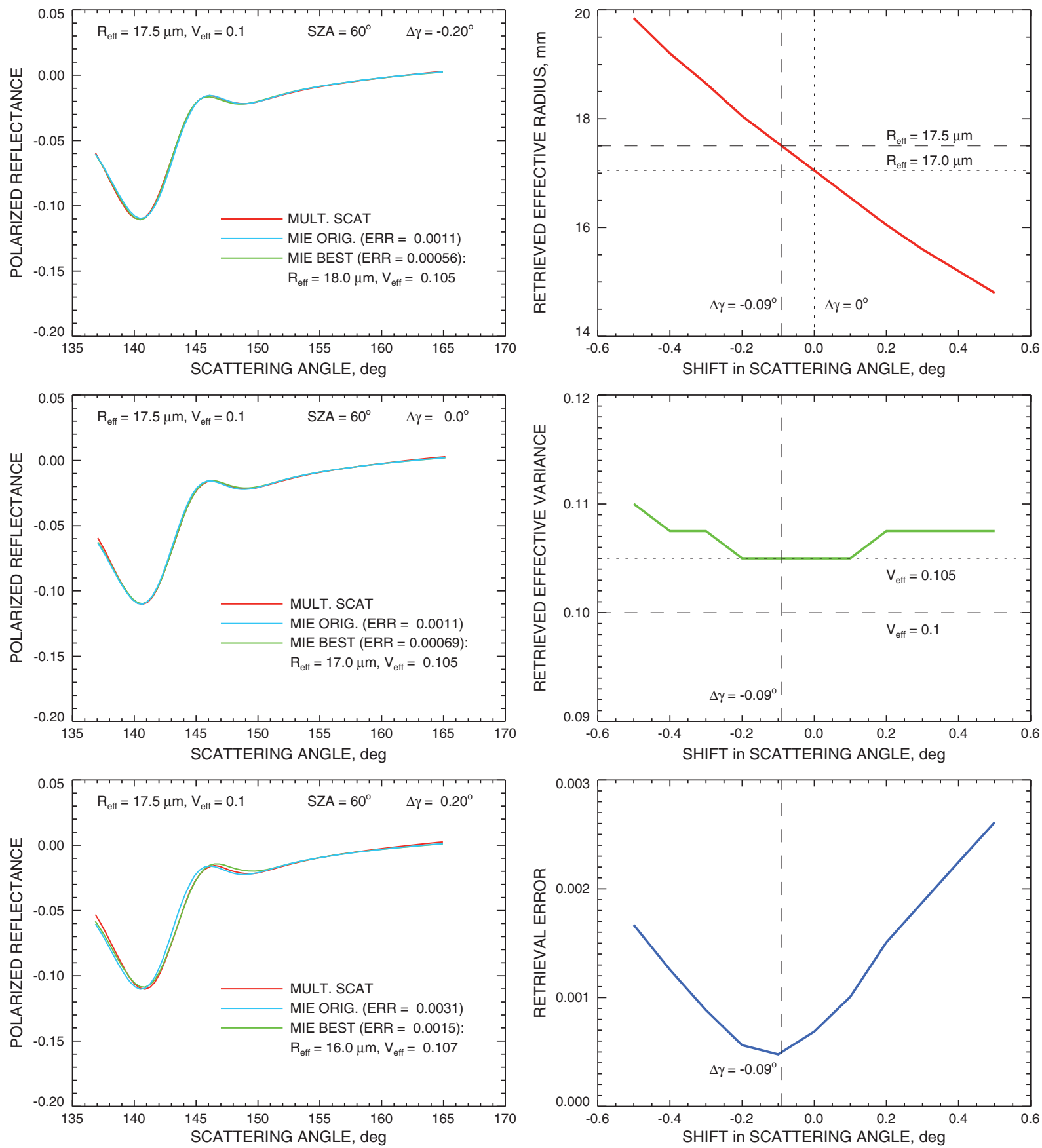

Fig. 8. Left: Polarized reflectances and retrievals from simulated RSP measurements with artificial scattering angle shifts: $\Delta \gamma=-0.2^{\circ}$ (top), $0^{\circ}$ (middle), and $0.2^{\circ}$ (bottom). In all cases $r_{\text {teff }}=17.5 \mu \mathrm{m}, v_{\text {eff }}=0.1, \mathrm{COD}=5$, SZA is $60^{\circ}$, and the relative solar-aircraft azimuth is $0^{\circ}$. Right: Sensitivity of the retrievals from simulated RSP measurements to scattering angle shifts ranging from $-0.5^{\circ}$ to $0.5^{\circ}$.

distribution. The average extinction efficiency $<Q_{\text {ext }}>$ is defined by Hansen and Travis (1974) as

$\left\langle Q_{\text {ext }}\right\rangle=\frac{\left\langle\sigma_{\text {ext }}\right\rangle}{G}=\frac{\left\langle\pi r^{2} Q_{\text {ext }}(r)\right\rangle}{\left\langle\pi r^{2}\right\rangle}$,

where $G$ is the average geometric cross-section. It follows from Eq. (16) that

$\frac{\mathrm{d} N_{\mathrm{c}}}{\mathrm{d} \tau}=\frac{1}{\pi\left\langle Q_{\mathrm{ext}}\right\rangle_{\tau}\left\langle r^{2}\right\rangle_{\tau}}$,

where $<r^{2}>_{\tau}$ is the second moment of the particle size distribution $n_{\tau}(r)$. Note, that for large cloud particles $<Q_{\text {ext }}>\approx 2$ regardless of the specific droplet size. If particle radius is measured in microns, the units of the concentration $N_{c}$ will be $\mu \mathrm{m}^{-2}$.

While the distribution $n_{\text {top }}(r)$ can have an arbitrary functional shape, we can estimate its effective radius

$r_{\text {eff }}^{\text {(top) }}=\frac{\left\langle r^{3}\right\rangle_{\text {top }}}{\left\langle r^{2}\right\rangle_{\text {top }}}$,

and variance

$v_{\text {eff }}^{\text {(top })}=\frac{\left\langle r^{4}\right\rangle_{\text {top }}\left\langle r^{2}\right\rangle_{\text {top }}}{\left\langle r^{3}\right\rangle_{\text {top }}^{2}}-1$, 

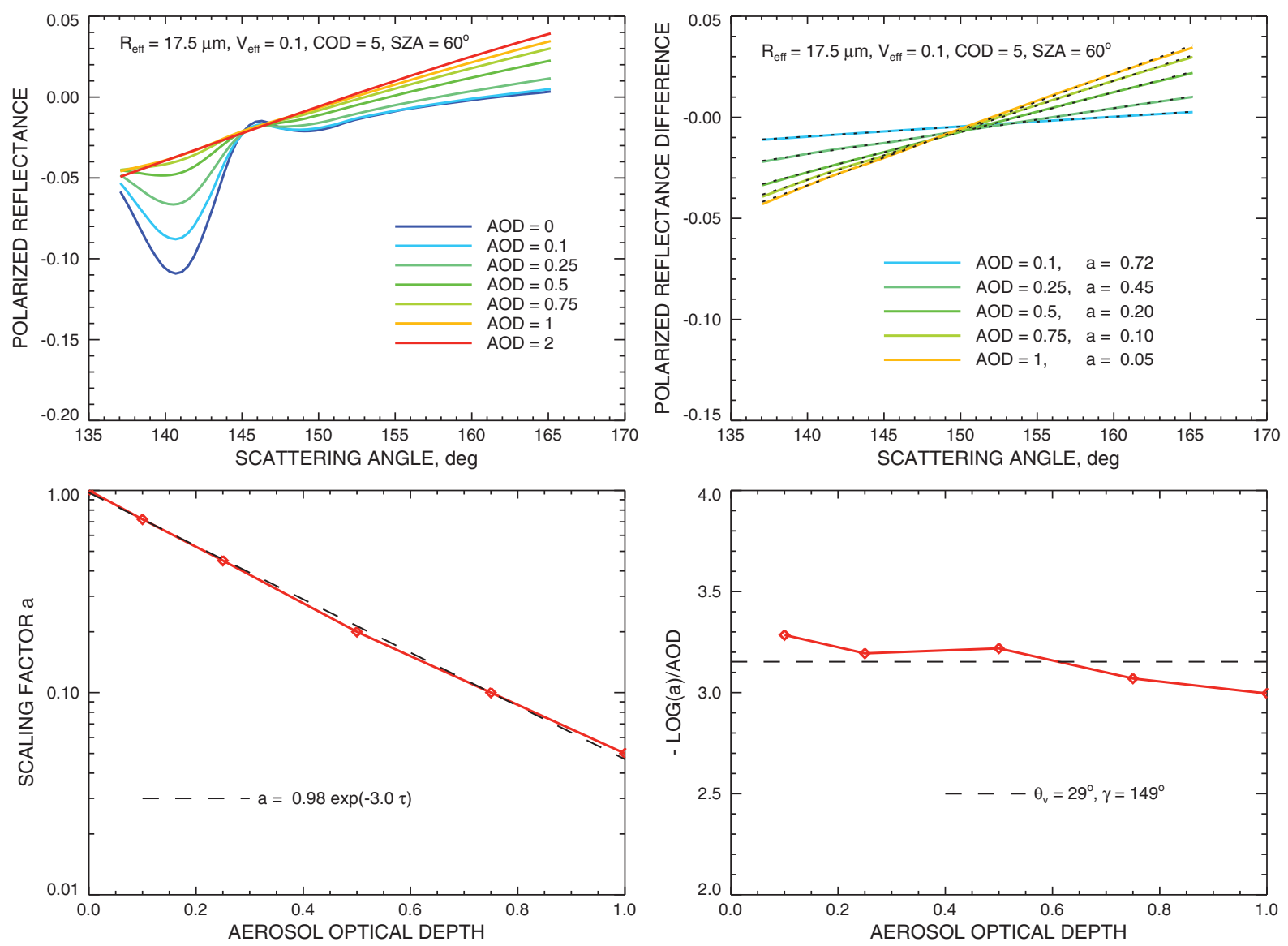

Fig. 9. Summary of the retrievals from the mixed atmosphere simulations. Top left: polarized reflectances vs. scattering angle for a variety of the top layer AOD. Top right: the residues of the reflectances from top left panel remaining after subtraction of the (scaled with the fitting factor $a$ ) reflectance corresponding to AOD $=0$ (solid lines), and linear fits to these residues (dashed lines). Bottom left: dependence of the fitting factor $a$ on AOD. Bottom right: inversion of the mean scattering angle from this dependence.

which are the primary determinants of the cloud's optical properties (cf. Hansen \& Travis, 1974). The moments of the distribution (11) are

$\left\langle r^{\mathrm{m}}\right\rangle_{\text {top }}=\int_{0}^{\infty} r^{\mathrm{m}} n_{\text {top }}(r) \mathrm{d} r=\int_{0}^{\tau_{\max }}\left\langle r^{\mathrm{m}}\right\rangle_{\tau} w(\tau) \mathrm{d} \tau$,

where $<r^{\mathrm{m}}>_{\tau}$ are the corresponding moments of the distribution $n_{\tau}(r)$.

Let us assume for simplicity that $n_{\tau}(r)$ has gamma distribution shape (cf. Hansen \& Travis, 1974)

$n(r)=\frac{(a b)^{(2 b-1) / b}}{\Gamma[(1-2 b) / b]} r^{(1-3 b) / b} e^{-r / a b}$

for every $\tau$, and that only $r_{\text {eff }}$ (parameter $a$ of gamma distribution) depends on $\tau$, while $v_{\text {eff }}$ (parameter $b$ ) is constant for the whole profile. Then

$\left\langle r^{\mathrm{m}}\right\rangle_{\text {top }}=\frac{b^{\mathrm{m}} \Gamma[(1 / b)-2+m]}{\Gamma[(1 / b)-2]} \overline{a^{\mathrm{m}}}$,

where

$\overline{a^{\mathrm{m}}}=\int_{0}^{\tau_{\max }} a^{\mathrm{m}}(\tau) w(\tau) \mathrm{d} \tau$, and a straight-forward calculation shows that

$r_{\text {eff }}^{\text {(top) }}=\frac{\left\langle r^{3}\right\rangle_{\text {top }}}{\left\langle r^{2}\right\rangle_{\text {top }}}=\frac{\overline{a^{3}}}{\overline{a^{2}}}$

and

$v_{\mathrm{eff}}^{\text {(top) }}=\frac{\left\langle r^{4}\right\rangle_{\text {top }}}{\left\langle r^{2}\right\rangle_{\text {top }}\left[r_{\text {eff }}^{\text {(top) }}\right]^{2}}-1=(1+b) \frac{\overline{a^{4}} \overline{a^{2}}}{\left[\overline{\left.a^{3}\right]^{2}}\right.}-1$.

\subsection{2-layer cloud}

Let us consider the simplest case of a stratified cloud consisting of two layers. Let the top and bottom layers have droplet size distributions with common $v_{\text {eff }}=b$ and different $r_{\text {eff, }}$, respectively $a_{1}$ and $a_{2}$ (constant within each layer). The top layer has optical depth $\tau_{1}$.

\subsubsection{Effective radius and variance}

In the 2-layer cloud case the $w$-moments (24) will have the form 

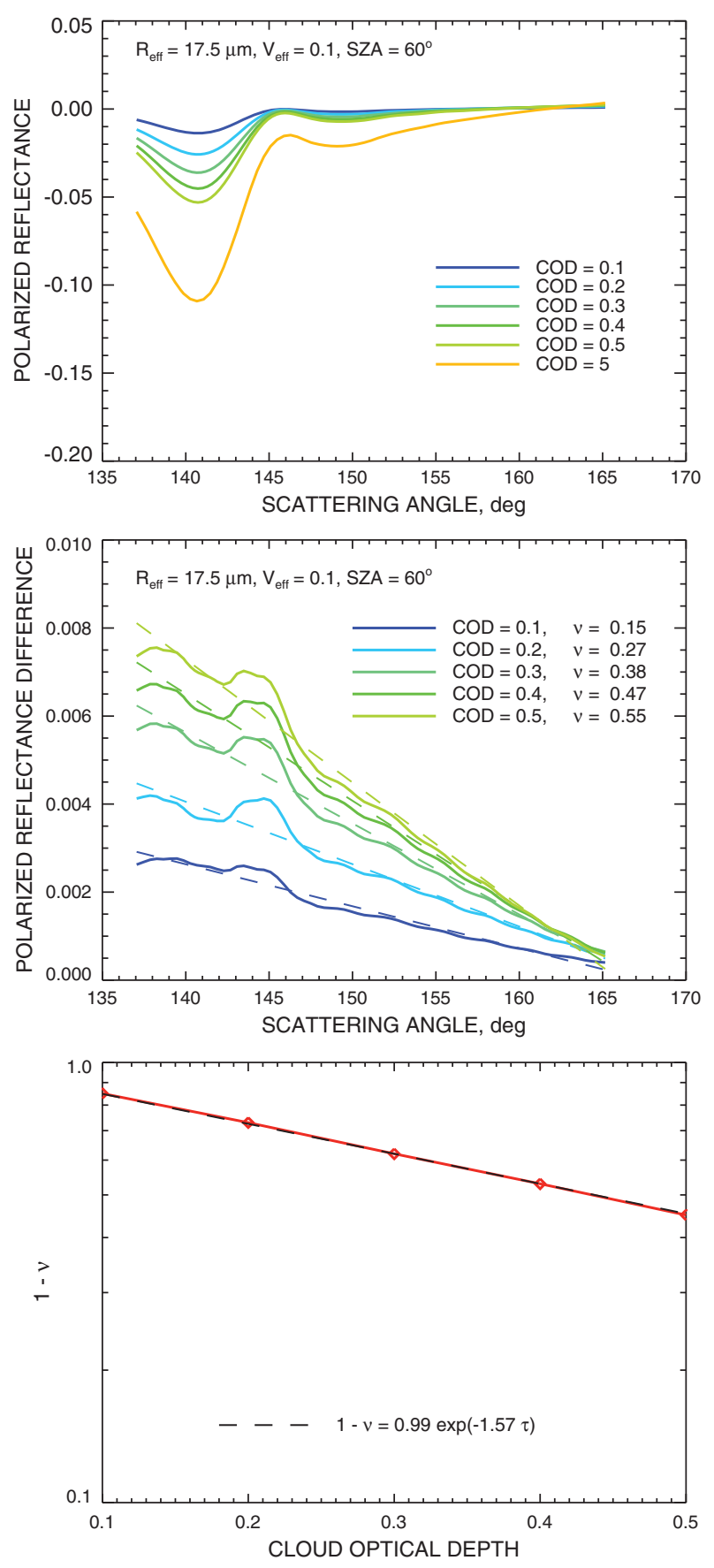

Fig. 10. Summary of single layer simulations for $r_{\text {eff }}=17.5 \mu \mathrm{m}$ and $v_{\text {eff }}=0.1$. Top: polarized reflectances vs. scattering angle for a variety of the layer optical depths. Middle: the residues of the reflectances from top left panel remaining after subtraction of the (scaled with the fitting factor $v$ ) reflectance corresponding to $\tau=5 \simeq \infty$ (solid lines), and linear fits to these residues (dashed lines). Bottom right: fit of $u=(1-v)$ by an exponential function of $\tau$.

Using the normalization condition (12), this expression can be written as

$\overline{a^{\mathrm{m}}}=\left(a_{1}^{\mathrm{m}}-a_{2}^{\mathrm{m}}\right) W+a_{2}^{\mathrm{m}}$,

where we introduced the notation of the top layer weight

$W=\int_{0}^{\tau_{1}} w(\tau) \mathrm{d} \tau$ which can take values between 0 and 1 . Note that the function $w(\tau)$ can be different for different $\tau_{1}$ (i.e., $w=w\left(\tau_{1} ; \tau\right)$, thus, generally it is not a derivative of $W\left(\tau_{1}\right)$ with respect to $\left.\tau_{1}\right)$. As follows from Eqs. (25) and (28), $r_{\text {eff }}{ }^{\text {top })}$ can be expressed through $W$ as

$r_{\text {eff }}^{\text {(top) }}=\frac{\left(a_{1}^{3}-a_{2}^{3}\right) W+a_{2}^{3}}{\left(a_{1}^{2}-a_{2}^{2}\right) W+a_{2}^{2}}$.

Note, that if $a_{1}=a_{2}$, the dependence on $\tau_{1}$ disappears, as expected. If the top layer is thin $(W \ll 1)$, we retrieve $r_{\text {eff }}^{\text {(top })} \approx a_{2}$, while $r_{\text {eff }}^{\text {(top })} \approx a_{1}$ when it is thick $(W \approx 1)$. The weight $W$ can be expressed using Eq. (30) in terms of the values of $a_{1}, a_{2}$, and $r_{\text {eff }}^{\text {(top) }}$, and then substituted back into Eqs. (28) and (26) to provide the relationship between the observed effective variance and the radius:

$v_{\mathrm{eff}}^{\text {(top) }}=b-(1+b) \delta_{1} \delta_{2}$,

where

$\delta_{i}=\frac{r_{\mathrm{eff}}^{\text {(top) }}-a_{i}}{r_{\mathrm{eff}}^{\text {(top) }}}$

for $i=1,2$ (see Appendix A for details of these calculations). Note, that since $r_{\text {eff }}^{\text {(top) }}$ is always in between $a_{1}$ and $a_{2}, \delta_{1}$ and $\delta_{2}$ have the opposite signs. Thus, their product lta ${ }_{1} \delta_{2} \leq 0$, and consequently $v_{\text {eff }}^{(\text {top })} \geq b$. Note that $v_{\mathrm{eff}}^{\text {(top) }}=b$ in the limit case of very thick or very thin top

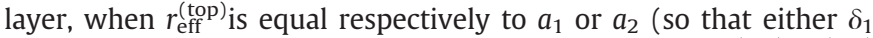
or $\delta_{2}$ is zero). As a function of $\tau_{1}$ (or, equivalently, of $r_{\text {eff }}^{\text {(top) }}$ ) $v_{\text {eff }}^{\text {(top) }}$ has its maximum value when $r_{\text {eff }}^{\text {(top) }}$ is equal to the harmonic mean of $a_{1}$ and $a_{2}$ :

$\frac{1}{r_{\mathrm{eff}}^{\text {(top) }}}=\frac{1}{2}\left(\frac{1}{a_{1}}+\frac{1}{a_{2}}\right)$

The maximal value of $v_{\mathrm{eff}}^{(\mathrm{top})}$ is then

$\max v_{\mathrm{eff}}^{\text {(top) }}=b+(1+b) \frac{\left(a_{2}-a_{1}\right)^{2}}{4 a_{1} a_{2}}$.

\subsubsection{Exponential weighting function}

The results presented so far are valid for weighting function $w(\tau)$ of any shape. Let us now introduce a specific weighting function for a 2-layer cloud following Eq. (14):

$w(\tau)=C \cdot\left\{\begin{array}{cc}s_{1} e^{-\beta_{1} \tau}, & \tau \leq \tau_{1} \\ s_{2} e^{-\beta_{1} \tau_{1}} e^{-\beta_{2}\left(\tau-\tau_{1}\right)}, & \tau_{1}<\tau \leq \tau_{\max }\end{array}\right.$,

where $C$ is the normalization constant, and $s_{1}$ and $s_{2}$ reflect the relationship between droplet number concentration and optical depth (depending on particle size). Note that for gamma distribution

$\frac{\mathrm{d} N_{\mathrm{c}}}{\mathrm{d} \tau}=\left[\pi\left\langle\mathrm{Q}_{\mathrm{ext}}\right\rangle a^{2}(1-b)(1-2 b)\right]^{-1}$,

and if we assume that $\left\langle Q_{\mathrm{ext}}>\approx 2\right.$ is the same for the two layers and so is the effective variance $b$, we can set

$s_{1}=\frac{1}{a_{1}^{2}}$, and $s_{2}=\frac{1}{a_{2}^{2}}$.

(Note, that the exact values of $\left\langle Q_{\text {ext }}\right\rangle$ can be incorporated by rescaling: $a \rightarrow<Q_{\text {ext }}>^{1 / 2}$ a.) It is convenient to introduce the 
notations $u_{1}$ and $u_{2}$ respectively for the top and bottom layer attenuation functions:

$$
\begin{aligned}
& u_{1}=\exp \left(-\beta_{1} \tau_{1}\right), \\
& u_{2}=\exp \left[-\beta_{2}\left(\tau_{\max }-\tau_{1}\right)\right] .
\end{aligned}
$$

Using this notation

$$
C=\left\{\frac{s_{1}}{\beta_{1}}+\left[\frac{s_{2}}{\beta_{2}}\left(1-u_{2}\right)-\frac{s_{1}}{\beta_{1}}\right] u_{1}\right\}^{-1},
$$

and therefore,

$W\left(\tau_{1}\right)=\frac{1-u_{1}}{1+(\xi-1) u_{1}}$,

where

$\xi=\frac{\beta_{1}}{s_{1}} \cdot \frac{s_{2}}{\beta_{2}}\left(1-u_{2}\right)=\frac{\beta_{1}}{\beta_{2}} \cdot \frac{a_{1}^{2}}{a_{2}^{2}}\left(1-u_{2}\right)$

Substitution of Eq. (41) into Eq. (30) yields the following expression for the effective radius:

$r_{\text {eff }}^{\text {(top) }}=a_{1}+\frac{\left(\frac{a_{2}}{a_{1}}\right)^{2} \xi \cdot\left(a_{2}-a_{1}\right) e^{-\beta_{1} \tau_{1}}}{1+\left[\left(\frac{a_{2}}{a_{1}}\right)^{2} \xi-1\right] e^{-\beta_{1} \tau_{1}}}$.

Note that if $\tau_{1}=\tau_{\max }$ then $\xi=0$, and $r_{\text {eff }}^{\text {(top) }}=a_{1}$. If $\tau_{1}=0$, then $\exp \left(-\beta_{1} \tau_{1}\right)=1$, and $r_{\text {eff }}^{\text {top })}=a_{2}$. If the coefficients $s_{i}$ are specified according to Eq. (37) and $\beta_{1}=\beta_{2}=\beta$, then Eq. (43) takes the form

$r_{\mathrm{eff}}^{\text {(top) }}=a_{1}+\frac{e^{-\beta \tau_{1}}-e^{-\beta \tau_{\max }}}{1-e^{-\beta \tau_{\max }}}\left(a_{2}-a_{1}\right)$

which is especially simple in the case of semi-infinite medium:

$r_{\text {eff }}^{\text {(top) }}=a_{1}+e^{-\beta \tau_{1}}\left(a_{2}-a_{1}\right)$.

The 2-layer distribution (35) can be naturally generalized to the case of a continuous profile, characterized by the dependences of $s=r_{\text {eff }}^{2}$ and the attenuation coefficient $\beta$ on the optical depth $\tau$ :

$w(\tau)=C s(\tau) \exp \left[-\int_{0}^{\tau} \beta(\tau) \tau \mathrm{d} \tau\right]$,

where the normalization constant $C$ is determined from the condition (12).

\subsubsection{Numerical results}

We conducted a series of numerical tests involving 2-layer clouds. In these tests simulated RSP measurements were made in the principal plane for a SZA of $60^{\circ}$. In all simulations the total optical depth of the 2-layer cloud was 5 (that is practically infinite for polarized reflectance), while the top layer COD took the values of $0,0.1,0.2,0.3$, $0.4,0.5,0.7,1,1.5,2.5,4$, and 5 . The layers had different $r_{\text {eff }}$ ( 7.5 and $17.5 \mu \mathrm{m}$ ) and the same $v_{\text {eff }}=0.1$. Two series of tests were conducted. In the first series ("Test A," Fig. 11 (left)) the layer with $17.5 \mu \mathrm{m}$ droplets was on top, while in the second series ("Test B", Fig. 11 (right)) the layer with smaller $7.5 \mu \mathrm{m}$ droplets was on top. The RSP-type retrievals of $r_{\text {eff }}^{(\text {top) }}$ and $v_{\text {eff }}^{(\text {top })}$ are summarized in Table 1 and Fig. 12 (in the Figure, the nominal values of $r_{\text {eff }}$ and $v_{\text {eff }}$ were used instead of retrievals at large $\tau_{1}$ and at $\tau_{1}=0$ ). The maximum value of $v_{\text {eff }}$ allowed in the retrievals was 0.35. The top left plot in Fig. 12 shows that, as the top layer optical depth increases to $\tau_{1} \sim 1-1.5$, the retrieved value of $r_{\text {eff }}^{\text {(top) }}$ reaches its limit value, which would be retrieved in the absence of the bottom layer. This convergence is apparently slower in Test A, where the top layer consists of larger particles. This means that even at the same optical depth the top layer with larger particles is more transparent than that with smaller ones, allowing for stronger influence of the bottom layer composition on the retrieved droplet size. This is consistent with the conjecture that small-angle multiple forward scattering events contribute to formation of the rainbow. Fig. 11 shows that even when the observed polarized reflectance is a mixture of contributions from the 2 layers, and the effective size distribution $n_{\text {top }}$ is essentially bimodal (e.g., at $\tau_{1}=0.5$ ), the Mie-theory-based LUT assuming a monomodal gamma distribution can still provide an adequate fit to the results, although a larger effective variance is retrieved. The bottom plots in Fig. 12 show that the width of the observed size distribution first increases with the increase of the top layer optical depth (since now both top and bottom layers contribute to the observations), and then decreases as the influence of the bottom layer diminishes by $\tau_{1} \sim 1.5$. The dotted lines in this plot show the values of $v_{\text {eff }}^{\text {(top) }}$ reconstructed from the corresponding $r_{\text {eff }}^{\text {(top) }}$ series using Eq. (31), which are in a reasonably good agreement with the direct numerical results. The theoretical maximum of $v_{\mathrm{eff}}^{\text {protect(top) }}$, is 0.31 according to Eq. (34). It occurs (see Eq. (33)) at $r_{\text {eff }}^{\text {(top) }}=10.5 \mu \mathrm{m}$, that, corresponds to $\tau_{1}=0.34$ for Test $\mathrm{A}$, and to $\tau_{1}=0.41$ for Test $\mathrm{B}$. We see that, as we already pointed out above, our direct retrievals of $v_{\text {eff }}$ show a high bias. However, the positions of the maxima of the apparent effective variance in the direct retrievals are within 0.1 in optical depth of their theoretical estimates.

Comparisons between analytical estimates and numerical results from Fig. 12 are presented in Fig. 13. The plots in this Figure compare the dependency on the top layer optical depth of the analytical estimates for the retrieved droplet effective radius $r_{\mathrm{eff}}^{\text {(top) }}$, the retrieved effective variance and the top layer weights $W\left(\tau_{1}\right)$ with their numerical values obtained using Eq. (A.1). The bottom right plot in Fig. 13 shows the relationship between numerical and analytical values of the large $(17.5 \mu \mathrm{m})$ mode weight from the Tests A and B combined. While the values of the above described parameters retrieved from simulated data are in general agreement with the analytical predictions, certain systematic differences are clearly observed in Fig. 13. In particular, the numerical retrievals of $r_{\mathrm{eff}}^{(\mathrm{top})}$ show slower than predicted change in the retrieved $r_{\text {eff }}^{\text {(top) }}$ with increase of the top layer COD when the latter is small, and faster saturation when it is large. Also, the retrieved maximal values of $v_{\text {eff }}^{\text {(top) }}$ are larger than predicted and occur at different top layer CODs. These discrepancies are consistent with the errors associated with fitting the results generated with a bi-modal size distribution using LUT computed assuming that the distribution is mono-modal. Fig. 14 demonstrates these effects on fitting of both single-scattering polarized reflectances (left panels), and the size distribution shapes themselves (right panels). The retrievals here are presented as functions of the large $(17.5 \mu \mathrm{m})$ mode statistical weight, thus, the plots of $r_{\text {eff }}^{\text {(top) }}$ and $v_{\text {eff }}^{\text {(top) }}$ are similar to those in Fig. 13 corresponding to Test A. The bottom plots in Fig. 14 directly correspond to the bottom right plot in Fig. 13. All these plots show that least square fit algorithms tend to retrieve the parameters of the dominant mode in the bi-modal distribution rather than the averages over the whole distribution (that were computed analytically in these tests).

\section{Tests on 3D Monte Carlo simulations}

We used Monte Carlo simulations to study the influence of 3D radiative effects on RSP-type droplet size distribution retrievals. The radiative transfer model MYSTIC (Monte Carlo code for the phYSically correct Tracing of photons In Cloudy atmospheres; Mayer (2009)) was used for the simulation of RSP measurements for a 3D cloud field. MYSTIC is operated as one of several radiative transfer solvers of the libRadtran radiative transfer package (Mayer \& Kylling, 2005). Originally, MYSTIC was developed as a forward tracing method for 

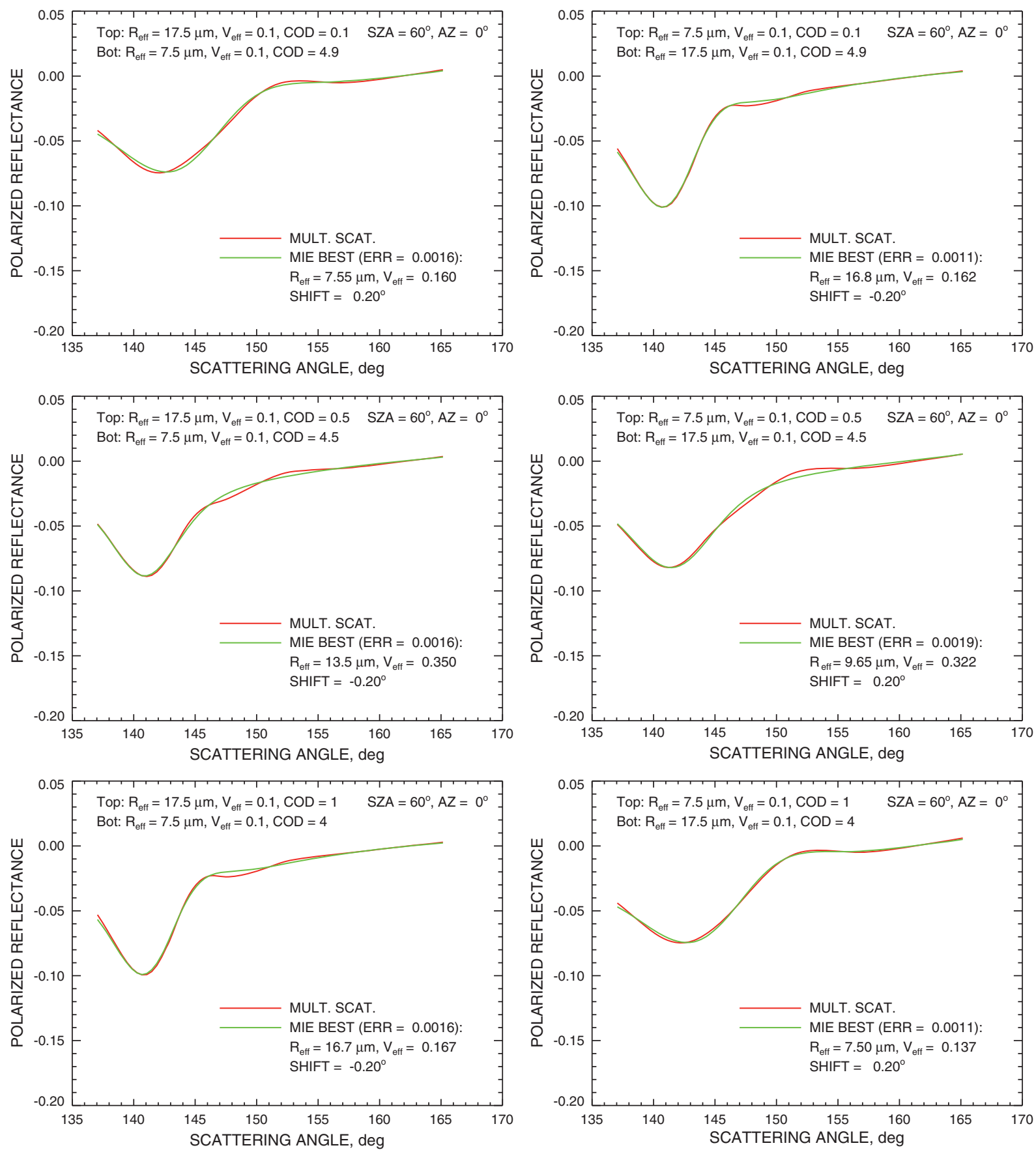

Fig. 11. RSP-type retrievals from simulated polarized reflectance of 2-layer cloud with total COD $=5$. Left: Test A (top layer $r_{\text {eff }}=17.5 \mu m$, bottom layer $r_{\text {eff }}=7.5 \mu$ m). Right: Test B (top layer $r_{\text {eff }}=7.5 \mu \mathrm{m}$, bottom layer $r_{\text {eff }}=17.5 \mu \mathrm{m}$ ). In both tests $v_{\text {eff }}=0.1$ for both layers. The top layer COD of is 0.1 (top), 0.5 (middle), and 1 (bottom). Red curves correspond to the actual multiple scattering simulations, while green curves show the best solution from the RSP retrieval algorithm.

the calculation of irradiances and radiances in 3D plane-parallel atmospheres. Recently, the model has been extended to include spherical geometry and a backward tracing mode (Emde \& Mayer, 2007). In addition the model has been extended by Emde et al. (2010) to include polarized radiation from scattering by randomly oriented particles, i.e. clouds, aerosols, and molecules. MYSTIC includes variance reduction methods (Buras \& Mayer, 2011) which are required for efficient unbiased radiative transfer simulations in cloudy atmospheres.

This model was applied to a realistic cloud field obtained from a large-eddy simulation (LES) of shallow, maritime convection. The LES model (Ackerman et al., 2004) treats three-dimensional fluid dynamics of the atmosphere and incorporates a bin microphysics model that resolves the size distributions of aerosol and cloud droplets in each grid cell. It also includes a two-stream radiative transfer model that treats the vertical transport of radiation in each model column. The LES dataset used in this study is based on idealizations of measurements obtained during the Rain in Cumulus over the Ocean project (RICO, vanZanten et al., 2011), which was chosen to maximize the likelihood of observing 3D cloud effects on retrieved droplet sizes. This simulation has $100 \mathrm{~m}$ horizontal and $40 \mathrm{~m}$ vertical resolution. To simplify the comparison between Monte Carlo computations and 1D RT model, the actual bin-by-bin LES size distributions in each horizontal layer were replaced by gamma distributions with the same $r_{\text {eff }}$, as in the microphysical model, and the effective variance 
Table 1

Results of RSP-type retrievals from simulated polarized reflectances of a 2-layer cloud with various top layer optical depth (total COD of both layers is 5). Test A: top layer $r_{\text {eff }}=17.5 \mu \mathrm{m}$, bottom layer $r_{\text {eff }}=7.5 \mu \mathrm{m}$; Test B: vice versa. In all cases $v_{\text {eff }}=0.1$ for both layers.

\begin{tabular}{|c|c|c|c|c|}
\hline \multirow[t]{3}{*}{ Top $\tau$} & \multicolumn{2}{|l|}{ Test A } & \multicolumn{2}{|l|}{ Test B } \\
\hline & \multicolumn{2}{|c|}{$\begin{array}{l}\text { Top: } r_{\text {eff }}=17.5 \mu \mathrm{m} \\
\text { Bot:: } r_{\text {eff }}=7.5 \mu \mathrm{m}\end{array}$} & \multicolumn{2}{|c|}{$\begin{array}{l}\text { Top: } r_{\text {eff }}=7.5 \mu \mathrm{m} \\
\text { Bot:: } r_{\text {eff }}=17.5 \mu \mathrm{m}\end{array}$} \\
\hline & $r_{\mathrm{eff}}^{\text {(top) }}, \mu \mathrm{m}$ & $v_{\text {eff }}^{(\text {top })}$ & $\overline{r_{\mathrm{eff}}^{\text {(top) }}, \mu \mathrm{m}}$ & $v_{\text {eff }}^{(\text {top })}$ \\
\hline 0 & 7.45 & 0.117 & 17.8 & 0.100 \\
\hline 0.1 & 7.55 & 0.160 & 16.8 & 0.162 \\
\hline 0.2 & 8.70 & 0.285 & 15.1 & 0.285 \\
\hline 0.3 & 9.90 & 0.350 & 12.2 & 0.350 \\
\hline 0.4 & 11.3 & 0.350 & 10.6 & 0.350 \\
\hline 0.5 & 13.5 & 0.350 & 9.65 & 0.322 \\
\hline 0.7 & 15.2 & 0.292 & 8.25 & 0.192 \\
\hline 1 & 16.7 & 0.167 & 7.50 & 0.137 \\
\hline 1.5 & 17.5 & 0.117 & 7.45 & 0.120 \\
\hline 2.5 & 17.8 & 0.102 & 7.50 & 0.120 \\
\hline 4 & 17.8 & 0.100 & 7.45 & 0.117 \\
\hline 5 & 17.8 & 0.100 & 7.45 & 0.117 \\
\hline
\end{tabular}

uniformly set to 0.1 . The simulations of RSP measurements were made assuming that the instrument is flown in the solar principal plane (coinciding with the $y$-axis of the LES grid) at $2.4 \mathrm{~km}$ above ground. The solar zenith angle used in the model run was $40^{\circ}$, the surface was modeled as a Lambertian reflector with a $5 \%$ albedo and the measurement wavelength was $554 \mathrm{~nm}$. The US standard atmosphere was used for the pressure and trace gas profiles.
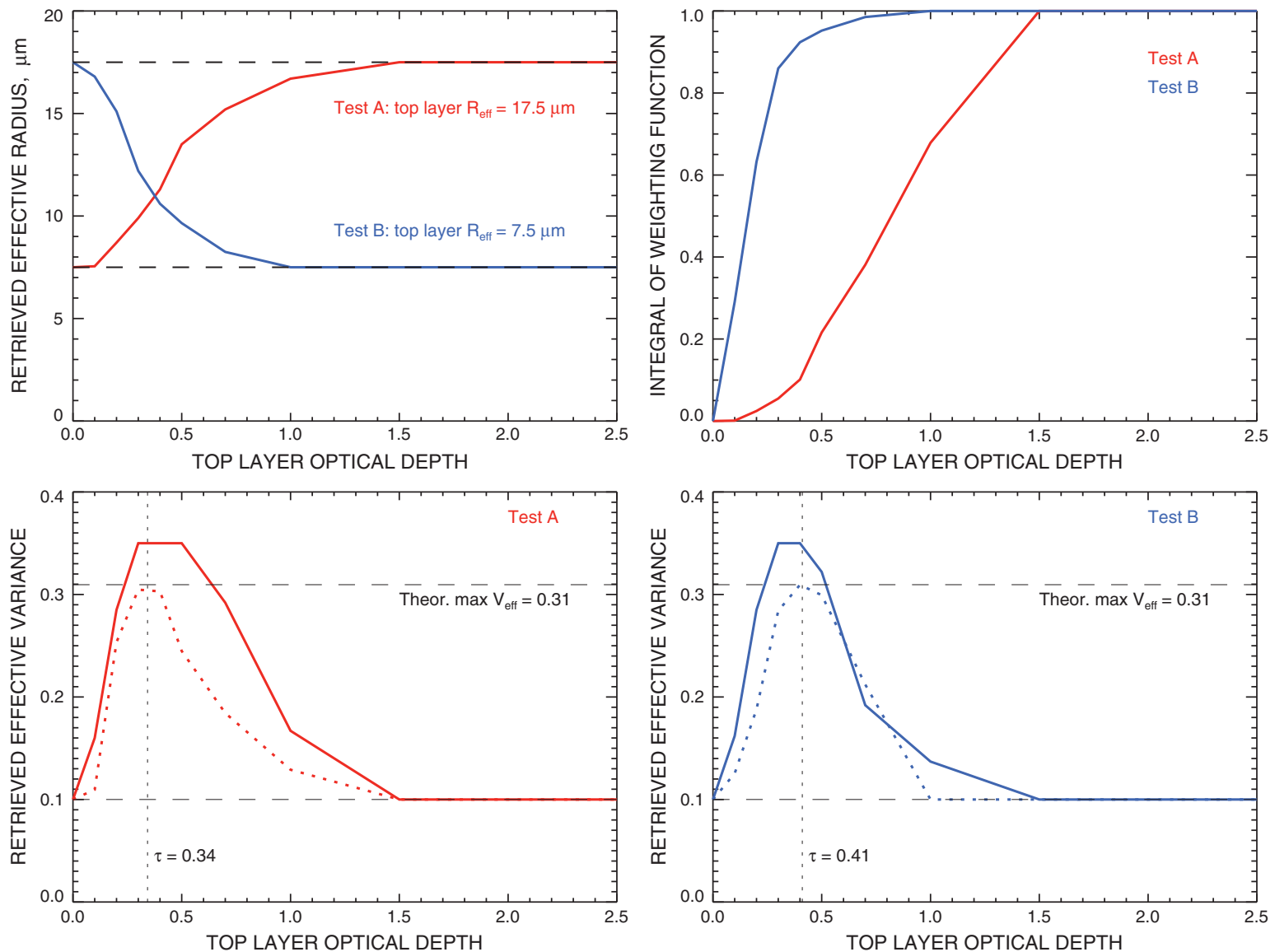

To evaluate the effect of the 3D nature of the radiative fields as opposed to the 1D vertical structure, we also simulated the corresponding reflected radiation using an independent pixel approximation (IPA) (Cahalan et al., 1994) applied to the vertical profiles of cloud droplet sizes and concentrations provided by the LES model. A total of 35 cloudy pixels (optical depth greater than 0.5) along two transect lines (corresponding to two RSP-like "trajectories") were originally selected for this comparison. The top left panel in Fig. 15 shows an example of the geometric shape of one of the cumulus clouds used. This shape is the cross-section along the transect line of points with droplet concentrations greater than $20 \mathrm{~cm}^{-3}$. The horizontal position of a "pixel" used for the comparison between $1 \mathrm{D}$ and $3 \mathrm{D}$ retrievals is depicted by the dashed line. The RSP reflectance simulated with the 3D RT model output was aggregated to the point where this dashed line crosses the cloud top boundary. The corresponding 1D simulations were made for a plane-parallel cloud with vertical profiles identical to those of the LES dataset along the dashed line. The profile of layer's $r_{\text {eff }}$ is shown in the left middle panel of Fig. 15, while the corresponding 1D and 3D simulated polarized reflectance is presented in the top right panel together with the results of RSP-type droplet size retrievals (the corresponding fits are depicted by dotted curves). The values of size distribution parameters retrieved for this pixel in the $1 \mathrm{D}$ and 3D cases are close: $r_{\mathrm{eff}}^{\left(\text {top }^{)}\right.}$ of respectively 13.2 and $13.5 \mu \mathrm{m}$, and $v_{\text {eff }}^{\text {(top) }}$ of respectively, 0.11 and 0.12 . These retrievals are consistent with $r_{\text {eff }}^{\text {(top })}=13.75 \mu \mathrm{m}$ computed directly from the LES model using a weighting function of the form given by Eq. (46).

It is seen from the top right panel of Fig. 15 that the magnitude of 3D polarized reflectance is substantially larger than that of its $1 \mathrm{D}$

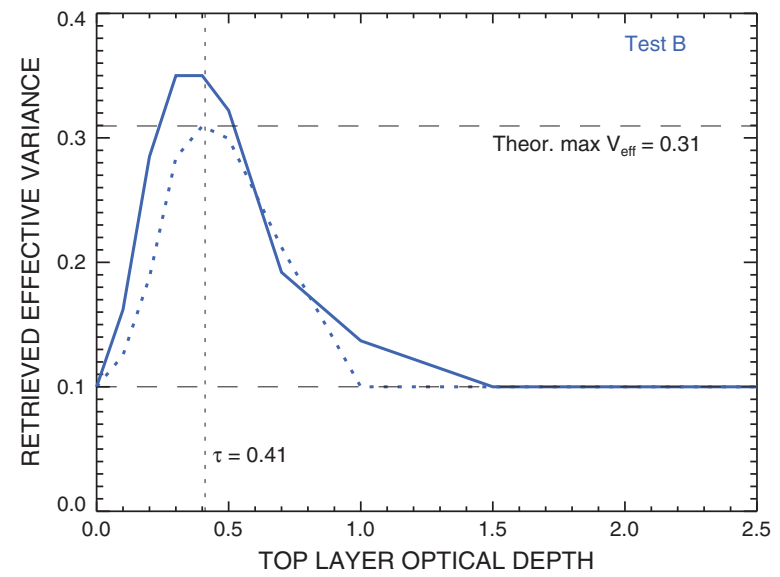

Fig. 12. Results of RSP retrievals made from simulated polarized reflectance of a 2-layer cloud. The data for Tests A and B are shown respectively in red and blue. Top left: dependence of the retrieved droplet effective radius $r_{\text {rotecteff }}^{\text {(top) }}$ on the top layer optical depth. The values of $r_{\text {eff }}$ for each single layer are shown by dashed lines. Top right: The top layer weights $W\left(\tau_{1}\right)$ (from Eq. (A.1)). Bottom: dependence of the retrieved effective variance on the top layer optical depth. Dashed curves depict the values of $v_{\text {eff }}^{\text {(top) }}$ computed from $r_{\text {eff }}^{\text {(top) }}$ according to Eq. (31). 

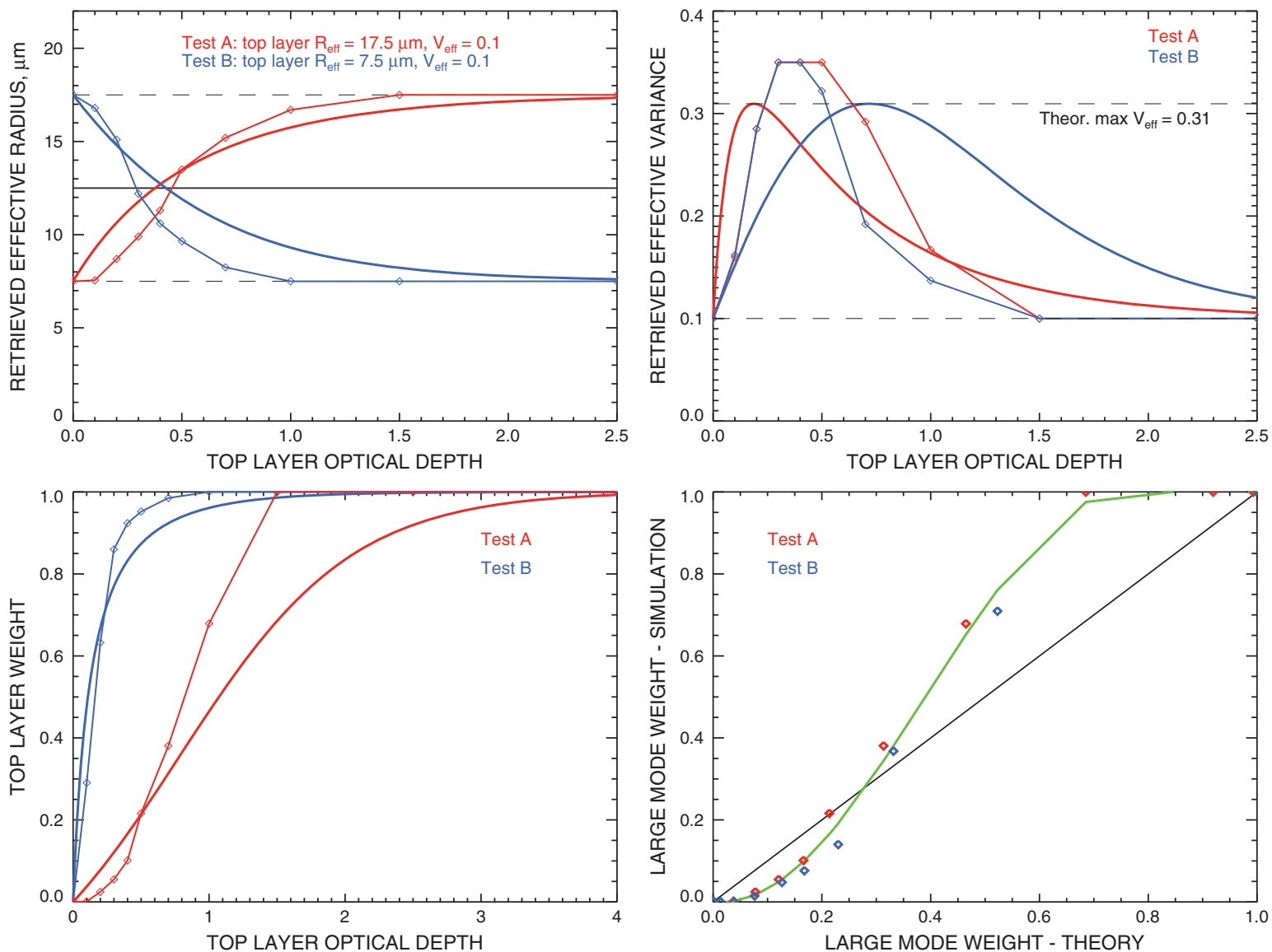

Fig. 13. Analytical estimations of the RSP retrievals in 2-layer cloud case (thick curves). The numerical results from Fig. 12 are shown by thin lines with diamonds depicting the actual data points. The data for Tests A and Bare shown respectively in red and blue. Top left: Dependence of the retrieved droplet effective radius $r_{\text {eff }}^{\text {(to })}$ on the top layer optical depth computed according to Eq. (30) with $W$ from Eq. (41) and attenuation coefficients from Section 8. Top right: Retrieved effective variance computed according to Eq. (31) as function of the top layer optical depth. Bottom left: The top layer weights $W\left(\tau_{1}\right)$ analytical values are from Eq. (41) , the numerical values are obtained using Eq. (A.1). Bottom right: relationship between numerical and analytical values of the large (17.5 $\mu \mathrm{m})$ mode weight from Tests A and B. Here the analytical weights are sampled at the data points of numerical simulations, the green curve represents a 5-degree polynomial fit to the numerical data.

analog. This enhancement is due to the contribution of light entering the cloud from its sides in the 3D case. However, this enhancement has an effect only on the pixels on bright sides of clouds, which are exposed to the direct sunlight. For the pixels on the shadowed side of the clouds the opposite takes place: 3D simulations produce a smaller reflectance than the corresponding plane-parallel models. Since the polarized reflectance on the shadowed side of the cloud is often too small to exhibit a rainbow structure, we restrict further 1D - 3D comparisons only to bright pixel cases. The middle right panel of Fig. 15 shows the histogram (for the set of all 35 cloudy pixels) of the ratio between 3D and 1D polarized reflectances (taken at their minimum around $141^{\circ}$ ), where the bright pixel cases are separated from those in shadow by a dashed line. We see that for the most part bright pixel 3D polarized reflectance exceeds its 1D counterpart by a factor of between 1.25 and 2 . The bottom plots present comparisons between effective radius and variance retrievals from the 1D and 3D radiative fields in 24 bright pixel cases. While no significant bias is seen between 1D and 3D values of $r_{\mathrm{eff}}^{\text {(top) }}$ ( $0.2 \mu \mathrm{m}$ mean difference and $0.73 \mu \mathrm{m}$ standard deviation of the difference), the corresponding $v_{\mathrm{eff}}^{\text {(top) }}$ values are systematically larger in the $3 \mathrm{D}$ case than in the $1 \mathrm{D}$ case. The $3 \mathrm{D}$ values range within $0.13 \pm$ 0.02 , while in the $1 \mathrm{D}$ case $v_{\text {eff }}^{(\text {top })}$ is close to 0.1 , which is the uniformly assumed single layer's $v_{\text {eff }}$ in the forward computations. This difference can be explained by the larger penetration depth of light into the cloud in 3D cases, which results from the previously noted illumination of the cloud from its sides. This effect causes a larger part of the non-uniform droplet size profile (cf. middle left panel) to contribute to the cloud-top retrievals, with a consequently wider droplet size distribution.

\section{Conclusions}

The presented sensitivity study of the accuracy of the cloud droplet size retrievals from the RSP measurements demonstrated that this technique is a potentially valuable tool for climate research.

Our algorithm retrieves the effective radius and variance of the cloud droplet size distribution, which is assumed to have a mono-modal gamma distribution shape. The tests were made on simulated datasets generated using both a plane-parallel (1D) and a Monte-Carlo (3D) RT model. We demonstrated that in the case of "measurements" made in the solar principle plane the accuracy of $r_{\text {eff }}$ retrievals is better on average than $0.15 \mu \mathrm{m}$ (with the worst recorded case of $7.1 \mu \mathrm{m}$ retrieval instead of $7.5 \mu \mathrm{m}$ at $v_{\text {eff }}=0.2$, that is a $5 \%$ error). Unlike $r_{\text {eff, }}$, the retrieved $v_{\text {eff }}$ shows systematic biases (see Fig. 4) ranging from $6 \%$ to $27 \%$ and generally decreasing with $r_{\text {eff. }}$. The largest absolute differences occur at large $v_{\text {eff. }}$ This discrepancy can be explained by the "smoothing" effect on the reflectance of multiple scattering, which is interpreted by the retrieval algorithm as a larger $v_{\text {eff. }}$ The droplet size retrievals in an arbitrary viewing geometry require the Stokes vector to be transformed from the measurement coordinate frame into that of the scattering plane. In this case $U$ is not identically zero and it is important to check that the parameter $Q$ resulting from this reference frame rotation contains all the single 

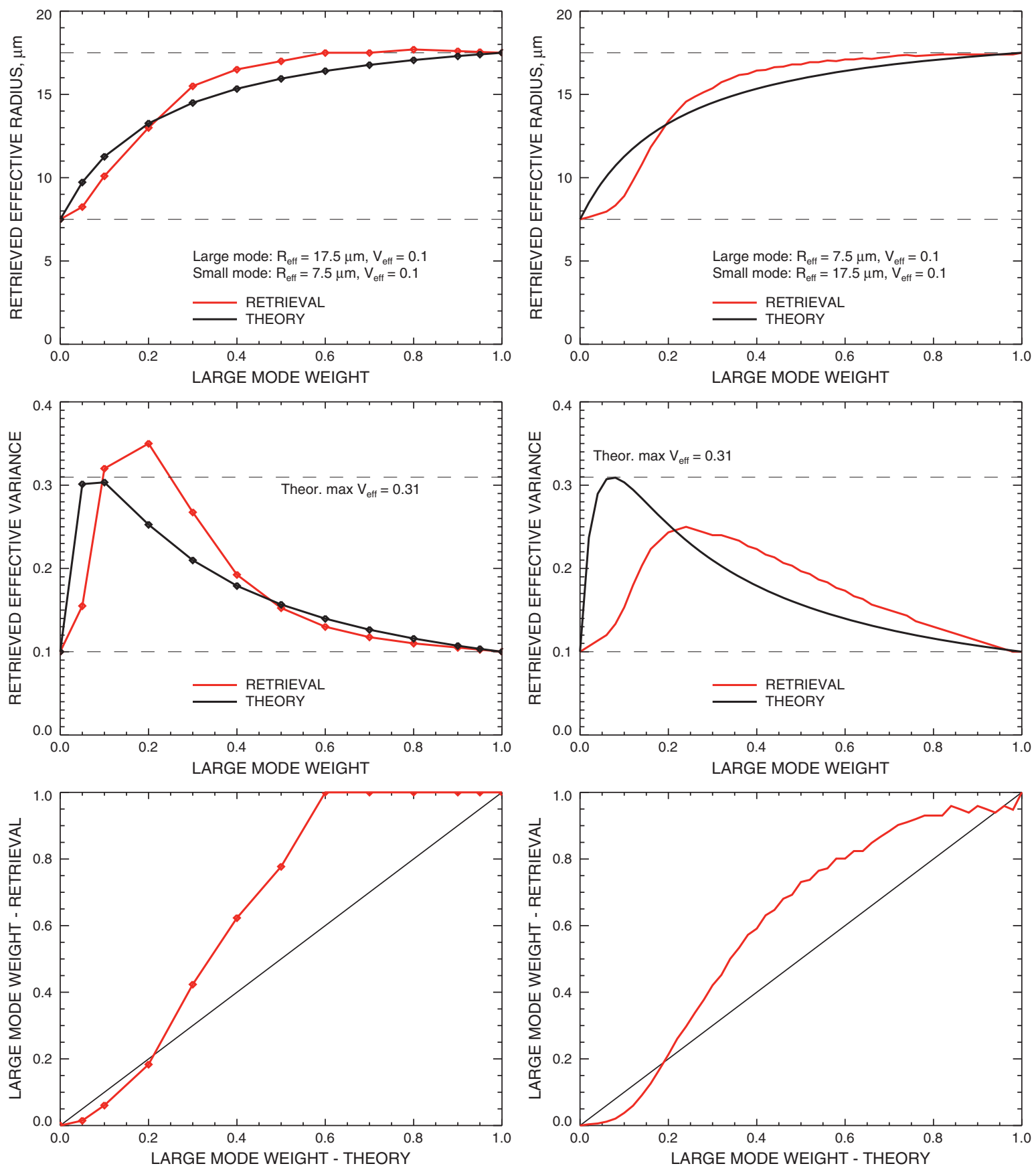

Fig. 14. Left: The effects of fitting of single-scattering polarized reflectance computed using Mie theory for bi-modal size distribution (with the same modes, as in Tests A and B) using LUT generated assuming mono-modal distribution model. Top: The retrieved effective radius (red) vs. the large (17.5 $\mu$ m) mode weight in the bi-modal distribution. Black curve represents the analytical values from Eq. (30). Middle: Same as top but for effective variance. Bottom: large mode weight derived from the fitted $r_{\text {eff }}$ (top plot) using Eq. (A.1) as function of the actual weight assumed in simulations. Right: same as left but for least square fit of size distributions themselves (rather than of derived optical parameters).

scattering information on which our retrieval is based. We find that the difference between retrievals from rotated datasets and their principle plane counterparts is negligible: as little as $0.1-0.2 \mu \mathrm{m}$ in radius and a few percentage points in variance. Our tests showed that uncertainties in scattering angle shift can be effectively resolved by considering the shift as a free parameter in the fitting procedure. This feature is implemented in our algorithm by introducing the shift parameter $\delta$ in Eq. (3). We also determined that the shape of the rainbow in polarized reflectance is unchanged by overlaying aerosol scattering, although the signal is attenuated according to Beer's law.

We demonstrated that the polarized rainbow amplitude includes contributions from both single and forward-directed multiple scattering.
While the latter does not affect the droplet size retrievals, an assessment of the relative magnitudes of the various contributions is instrumental in understanding from where in a stratified cloud the remotely sensed radiative properties are generated. Our tests performed on a family of 2-layer clouds with widely different effective radii demonstrated that the parameters retrieved from the simulated data are generally in agreement with the analytical predictions and show that for a typical airmass the retrieved size distribution parameters are generated within an optical depth of 1 from cloud top ( $50 \mathrm{~m}$ ). There are however certain systematic differences, which indicate that the least square fit algorithm used tends to output the parameters of the dominant mode rather than an average over the whole bi-modal distribution. This is particularly relevant to 

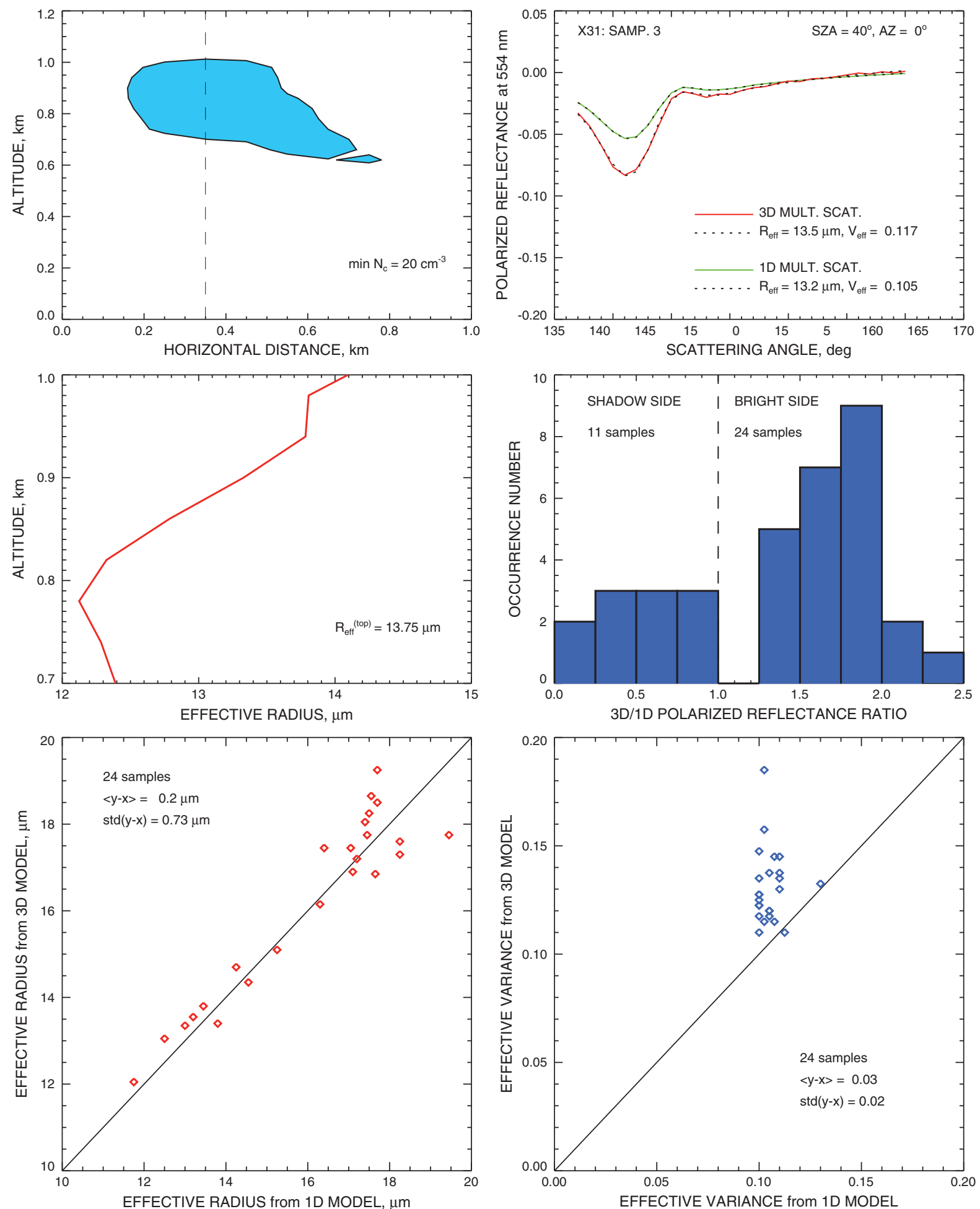

Fig. 15. Results of inter-comparison between droplet size retrievals from 1D and 3D simulated radiation fields. Top left: shape of a cumulus cloud vertical cross-section from LES output. Top right: polarized reflectances and RSP-type droplet size retrievals corresponding to the cloud section depicted by the dashed line in the top left plot. Middle left: effective radius profile corresponding to this dashed line (from microphysical model). Middle right: frequency histogram of the ratio between 3D and 1D polarized reflectances for 35 cloudy pixels. The dashed line separates parts of clouds illuminated by direct sunlight (bright cases) from those in shadow. Bottom left: comparison between effective radius values derived from 1D and 3D fields (bright cases only). Bottom right: same as bottom left but for the effective variance.

understanding under what conditions small evaporating drops near cloud top would be detected using polarization measurements. This problem may be potentially resolved by the Rainbow Fourier Transform (RFT), our new retrieval technique (Alexandrov et al., 2012) which allows for accurate retrieval of the size distribution shape of an arbitrary form (including multi-modal) with very good accuracy.
Our final tests of the retrieval algorithm were applied to 1D IPA and 3D simulations of the same cloud field consisting of shallow convective clouds representative of those observed during RICO. The fact that 3D cloud effects can increase, or decrease the magnitude of the rainbow structure means that using a tabulation of exact 1D RT results to reduce the bias in effective variance retrievals caused by 
multiple scattering effects is unlikely to be effective. This is because multiple scattering effects are inherently sensitive to 3D effects. Nonetheless we find that the effective radius retrievals from the 1D and 3D simulations agree extremely well while the effective variance retrieved from the $3 \mathrm{D}$ simulations is higher than that from the $1 \mathrm{D}$ simulations. The reason for this is that for the simulated viewing geometry (SZA $40^{\circ}$ in the principal plane) the rainbow is centered near nadir and side illumination of the clouds means that the rainbow is generated over a much larger depth into the clouds in the 3D simulations than in the 1D simulations. Vertical variations in effective radius are then interpreted as an increased effective variance. This result is not "wrong" but emphasizes the importance of understanding the vertical weighting associated with any particular droplet size retrieval method (Platnick, 2000). Our plans for the future include testing our algorithm on a simulated cloud fields that include thin ice clouds above water clouds (this situation is common for measurements from space or high-altitude aircraft).

In summary we find that the use of polarized reflectance observations of the rainbow to retrieve the effective radius is extremely robust against both aerosol and 3D effects. The retrieved effective variance tends to be biased high in 1D tests as a result of smoothing/ reduction in contrast of the rainbow structure caused by multiple scattering. The increased vertical depth over which the rainbow is generated in 3D clouds also tends to increase the retrieved effective variance. These biases are however relatively small and well understood and the retrieved effective variance is still a useful measure of droplet size dispersion near cloud top.

\section{Acknowledgments}

This research was funded by the NASA Radiation Sciences Program managed by Hal Maring and by the NASA Glory Mission project.

\section{Appendix A. Relationship between effective radius and variance in 2-layer cloud}

Eq. (30) can be used to express the top layer weight $W$ through the observed effective radius and those of the two layers:

$W=\frac{h_{2}}{h_{2}-h_{1}}$,

where

$h_{i}=a_{i}^{3}-a_{i}^{2} r_{\mathrm{eff}}^{\text {(top })}=a_{i}^{2}\left(a_{i}-r_{\mathrm{eff}}^{\text {(top })}\right)$

for $i=1,2$. In these notations the $w$-moments (28) take the form:

$$
\begin{aligned}
\overline{a^{\mathrm{m}}} & =\left(a_{1}^{\mathrm{m}}-a_{2}^{\mathrm{m}}\right) \frac{h_{2}}{h_{2}-h_{1}}+a_{2}^{\mathrm{m}} \\
& =\frac{a_{1}^{2} a_{2}^{2}}{h_{2}-h_{1}} \times\left[a_{1}^{\mathrm{m}-2} a_{2}-a_{1} a_{2}^{\mathrm{m}-2}-\left(a_{1}^{\mathrm{m}-2}-a_{2}^{\mathrm{m}-2}\right) r_{\text {eff }}^{\text {(top })}\right] .
\end{aligned}
$$

In particular,

$$
\begin{aligned}
\overline{a^{2}} & =\frac{a_{1}^{2} a_{2}^{2}}{h_{2}-h_{1}}\left(a_{2}-a_{1}\right), \\
\overline{a^{3}} & =\frac{a_{1}^{2} a_{2}^{2}}{h_{2}-h_{1}}\left(a_{2}-a_{1}\right) r_{\mathrm{eff}}^{\text {(top })}=\overline{a^{2}} r_{\mathrm{eff}}^{\text {(top) }} \\
\overline{a^{4}} & =\frac{a_{1}^{2} a_{2}^{2}}{h_{2}-h_{1}}\left(a_{2}-a_{1}\right)\left[\left(a_{1}+a_{2}\right) r_{\mathrm{eff}}^{\text {(top) }}-a_{1} a_{2}\right] \\
& \left.\left.=\frac{a^{2}}{\left(a_{1}\right.}+a_{2}\right) r_{\mathrm{eff}}^{\text {(top) }}-a_{1} a_{2}\right]
\end{aligned}
$$

When the expressions (A.4)-(A.6) are substituted into Eq. (26) for $v_{\text {eff }}^{\text {(top) }}$, the common factors $\overline{a^{2}}=a_{1}^{2} a_{2}^{2}\left(a_{2}-a_{1}\right) /\left(h_{2}-h_{1}\right)$ cancel from the ratio and we obtain

$v_{\mathrm{eff}}^{(\mathrm{top})}=(1+b) \frac{\left(a_{1}+a_{2}\right) r_{\mathrm{eff}}^{\text {(top) }}-a_{1} a_{2}}{\left(r_{\mathrm{eff}}^{\text {(top })}\right)^{2}}-1$

that can also be written in the form of Eq. (31).

\section{References}

Ackerman, A. S., Kirkpatrick, M. P., Stevens, D. E., \& Toon, O. B. (2004). The impact of humidity above stratiform clouds on indirect aerosol climate forcing. Nature, 432, 1014-1017.

Alexandrov, M. D., Cairns, B., \& Mishchenko, M. I. (in press). Rainbow Fourier transform. Journal of Quantitative Spectroscopy and Radiative Transfer, http://dx.doi.org/ 10.1016/j.jqsrt.2012.03.025.

Brenguier, J. -L., Pawlowska, H., \& Schüller, L. (2003). Cloud microphysical and radiative properties for parameterization and satellite monitoring of the indirect effect of aerosol on climate. Journal of Geophysical Research, 8632.

Bréon, F. M., \& Doutriaux-Boucher, M. (2005). A comparison of cloud droplet radii measured from space. IEEE Transactions on Geoscience and Remote Sensing, 1796-1805.

Bréon, F. -M., \& Goloub, P. (1998). Cloud droplet effective radius from spaceborne polarization measurements. Geophysical Research Letters, 1879-1882.

Buras, R., \& Mayer, B. (2011). Efficient unbiased variance reduction techniques for Monte Carlo simulations of radiative transfer in cloudy atmospheres: The solution. Journal of Quantitative Spectroscopy and Radiative Transfer, 112, 434-447.

C.-Labonnote, L., Brogniez, G., Doutriaux-Boucher, M., Buriez, J., Gayet, J., \& Chepfer, H. (2000). Modeling of light scattering in cirrus clouds with inhomogeneous hexagonal monocrystals. Comparison with in-situ and ADEOS-POLDER measurements. Geophysical Research Letters, 113-116.

Cahalan, R. F., Ridgway, W., Wiscombe, W., Gollmer, S., \& Harshvardhan (1994). Independent pixel and Monte Carlo estimates of stratocumulus albedo. Journal of Atmospheric Science, 3776-3790.

Cairns, B., Carlson, B. E., Lacis, A. A., \& Russell, E. E. (1997). An analysis of ground-based polarimetric sky radiance measurements. In D. H. Goldstein, \& R. A. Chipman (Eds.), Polarization: Measurement, Analysis, and Remote Sensing. Proc. SPIE, Vol. 3121. (pp. 382-393).

Cairns, B., Russell, E. E., \& Travis, L. D. (1999). Research scanning polarimeter: Calibration and ground-based measurements. In D. H. Goldstein, \& D. B. Chenault (Eds.), Polarization: Measurement, Analysis, and Remote Sensing. Proc. SPIE, Vol. 3754. (pp. 186-197).

Cairns, B., Waquet, F., Knobelspiesse, K., Chowdhary, J., \& Deuzé, J. L. (2009). Polarimetric remote sensing of aerosols over land surfaces. In A. A. Kokhanovsky, \& G. D. Leeuw (Eds.), Satellite aerosol remote sensing over land (pp. 295-325). Springer.

Chowdhary, J., Cairns, B., Mishchenko, M. I., Hobbs, P. V., Cota, G. F., Redemann, J., et al. (2005). Retrieval of aerosol scattering and absorption properties from photopolarimetric observations over the ocean during the CLAMS experiment. Journal of Atmospheric Science, 1093-1117.

Chowdhary, J., Cairns, B., Waquet, F., Knobelspiesse, K., Ottaviani, M., Redemann, J., et al. (2012). Sensitivity of multiangle, multispectral polarimetric remote sensing over open oceans to water-leaving radiance: Analyses of RSP data acquired during the MILAGRO campaign. Remote Sensing of Environment, 284-308.

Chowdhary, J., Mishchenko, M. I., \& Travis, L. D. (2006). Contribution of water-leaving radiances to multiangle, multispectral polarimetric observations over the open ocean: Bio-optical model results for case 1 waters. Applied Optics, 45, 5542-5567.

Coddington, O. M., Pilewskie, P., Redemann, J., Platnick, S., Russell, P. B., Schmidt, K. S., et al. (2010). Examining the impact of overlying aerosols on the retrieval of cloud optical properties from passive remote sensing. Journal of Geophysical Research, D10211.

de Haan, J. F., Bosma, P. B., \& Hovenier, J. W. (1987). The adding method for multiple scattering calculations of polarized light. Astronomy and Astrophysics, 371-382.

Deschamps, P. Y., Breon, F. M., Leroy, M., Podaire, A., Bricaud, A., Buriez, J. -C., et al. (1994). The POLDER mission - Instrument characteristics and scientific objectives. IEEE Transactions on Geoscience and Remote Sensing, 598-615.

Emde, C., Buras, R., Mayer, B., \& Blumthaler, M. (2010). The impact of aerosols on polarized sky radiance: Model development, validation, and applications. Atmospheric Chemistry and Physics, 383-396.

Emde, C., \& Mayer, B. (2007). Simulation of solar radiation during a total eclipse: A challenge for radiative transfer. Atmospheric Chemistry and Physics, 2259-2270.

Gilerson, A., Zhou, J., Oo, M., Chowdhary, J., Gross, B. M., Moshary, F., et al. (2006). Retrieval of chlorophyll fluorescence from reflectance spectra through polarization discrimination: Modeling and experiments. Applied Optics, 45, 5568-5581.

Girolamo, L. D., Liang, L., \& Platnick, S. (2010). A global view of one dimensional solar radiative transfer through oceanic water clouds. Geophysical Research Letters, L18809.

Goloub, P., Herman, M., Chepfer, H., Riedi, J., \& Brogniez, G. (2000). Cloud thermodynamical phase classification from the POLDER spaceborne instrument. Journal of Geophysical Research, 14747-14759.

Hansen, J. E., \& Travis, L. D. (1974). Light scattering in planetary atmospheres. Space Science Reviews, 527-610. 
Haywood, J. M., Osborne, S. R., \& Abel, S. J. (2004). The effect of overlying absorbing aerosol layers on remote sensing retrievals of cloud effective radius and cloud optical depth. Quarterly Journal of the Royal Meteorological Society, 779-800.

Hu, Y., Vaughan, M., McClain, C., Behrenfeld, M., Maring, H., Anderson, D., et al. (2007). Global statistics of liquid water content and effective number concentration of water clouds over ocean derived from combined CALIPSO and MODIS measurements. Atmospheric Chemistry and Physics, 3353-3359.

Knap, W. H., C.-Labonnote, L., Brogniez, G., \& Stammes, P. (2005). Modeling total and polarized reflectances of ice clouds: Evaluation by means of POLDER and ATSR-2 measurements. Applied Optics, 44, 4060-4073.

Knobelspiesse, K. D., Cairns, B., Ottaviani, M., Ferrare, R., Hair, J., Hostetler, C., et al. (2011). Combined retrievals of boreal forest fire aerosol properties with a polarimeter and lidar. Atmospheric Chemistry and Physics, 7045-7067.

Knobelspiesse, K. D., Cairns, B., Redemann, J., Bergstrom, R. W., \& Stohl, A. (2011) Simultaneous retrieval of aerosol and cloud properties during the Milagro field campaign. Atmospheric Chemistry and Physics, 6245-6263.

Knobelspiesse, K. D., Cairns, B., Schmid, B., Roman, M. O., \& Schaaf, C. B. (2008). Surface BRDF estimation from an aircraft compared to MODIS and ground estimates at the Southern Great Plains site. Journal of Geophysical Research, D20105.

Marshak, A., Platnick, S., Várnai, T., Wen, G., \& Cahalan, R. F. (2006). Impact of three-dimensional radiative effects on satellite retrievals of cloud droplet sizes. Journal of Geophysical Research, D09207.

Mayer, B. (2009). Radiative transfer in the cloudy atmosphere. European Physical Journal Conferences, 1, 75-99.

Mayer, B., \& Kylling, A. (2005). Technical note: The libRadtran software package for radiative transfer calculations description and examples of use. Atmospheric Chemistry and Physics, 1855-1877.

Mishchenko, M. I. (2006). Glory. In C. L. Parkinson, A. Ward, \& M. D. King (Eds.), Earth science reference handbook: A guide to NASA's earth science program and earth observing satellite missions (pp. 141-147). National Aeronautics and Space Administration.

Mishchenko, M. I., Cairns, B., Kopp, G., Schueler, C. F., Fafaul, B. A., Hansen, J. E., et al. (2007). Accurate monitoring of terrestrial aerosols and total solar irradiance: Introducing the Glory mission. Bulletin of the American Meteorological Society, 677-691.

Mishchenko, M. I., Travis, L. D., \& Lacis, A. A. (2006). Multiple scattering of light by par ticles: Radiative transfer and coherent backscattering. Cambridge University Press.

Nakajima, T., \& King, M. D. (1990). Determination of the optical thickness and effective particle radius of clouds from reflected solar radiation measurements. Part I: Theory. Journal of Atmospheric Science, 1878-1893.
Nakajima, T. Y., Suzuki, K., \& Stephens, G. L. (2010). Droplet growth in warm water clouds observed by the A-train. Part I: Sensitivity analysis of the MODIS-derived cloud droplet sizes. Journal of Atmospheric Science, 1884-1896.

Ottaviani, M., Cairns, B., Chowdhary, J., van Diedenhoven, B., Knobelspiesse, K. Hostetler, C., et al. (2012). Polarimetric retrievals of surface and cirrus clouds properties in the region affected by the Deepwater Horizon oil spill. Remote Sensing of Environment, 389-403.

Platnick, S. (2000). Vertical photon transport in cloud remote sensing problems. Journal of Geophysical Research, 22919-22935.

Platnick, S., King, M. D., Ackerman, S. A., Menzel, W. P., Baum, B. A., Riedi, J. C., et al. (2003). The MODIS cloud products: Algorithms and examples from Terra. IEEE Transactions on Geoscience and Remote Sensing, 459-473.

Platnick, S., \& Valero, F. P. J. (1995). A validation study of a satellite cloud retrieval during ASTEX. Journal of Atmospheric Science, 2985-3001.

van Diedenhoven, B., Cairns, B., Geogdzhayev, I. V., Fridlind, A. M., Ackerman, A. S. Yang, P., et al. (2012). Remote sensing of ice crystal asymmetry parameter using multi-directional polarization measurements. Part I: Methodology and evaluation with simulated measurements. Atmospheric Measurement Techniques Discussions 4321-4359.

van Diedenhoven, B., Fridlind, A., Ackerman, A., \& Cairns, B. (in press). Evaluation of hydrometeor phase and ice properties in cloud-resolving model simulations of tropical deep convection using radiance and polarization measurements. Journal of Atmospheric Sciences, http://dx.doi.org/10.1175/JAS-D-11-0314.1.

Vant-Hull, B., Marshak, A., Remer, L. A., \& Li, Z. (2007). The effects of scattering angle and cumulus cloud geometry on satellite retrievals of cloud drop effective radius. IEEE Transactions on Geoscience and Remote Sensing, 1039-1045.

vanZanten, M. C. Stevens, B. B. Nuijens, L., Siebesma, A. P. Ackerman, A., Burnet, F., et al. (2011). Controls on precipitation and cloudiness in simulations of trade-wind cumulus as observed during RICO. Journal of Advanced Modelling Earth System, M06001.

Waquet, F., Cairns, B., Knobelspiesse, K., Chowdhary, J., Travis, L. D., Schmid, B., et at (2009). Polarimetric remote sensing of aerosols over land. Journal of Geophysical Research, D01206.

Zinner, T., Wind, G., Platnick, S., \& Ackerman, A. S. (2010). Testing remote sensing on artificial observations: Impact of drizzle and 3-d cloud structure on effective radius retrievals. Atmospheric Chemistry and Physics, 9535-9549. 